\title{
Aufsätze
}

Thomas Zerback, Carsten Reinemann und Angela Nienierza

\section{Die Wahrnehmung des Meinungsklimas im Bundestagswahlkampf 2013}

Urteile über die Beliebtheit von Parteien und Erwartungen an ihr Abschneiden bei der Wahl

\section{Kurzfassung}

Wir beschäftigen uns mit dem Zustandekommen von Einschätzungen der wahrgenommenen Beliebtheit von Parteien (derzeitiges Meinungsklima) und den Erwartungen an ihr Abschneiden bei der Wahl (zukünftiges Meinungsklima). Auf Basis einer Befragung von Berliner Bürgern im Vorfeld der Bundestagswahl 2013 können wir für sieben deutsche Parteien zeigen, dass der wahrgenommene Medientenor sowie die im persönlichen Umfeld wahrgenommenen Meinungen die Einschätzungen des derzeit herrschenden Meinungsklimas gegenüber den Parteien maßgeblich prägen, wohingegen erinnerte Umfrageergebnisse kaum Einfluss ausüben. Mit Blick auf die Wahlerwartungen dominieren Umfragen allerdings die Einschätzungen der Bürger. Soziale Projektionen der eigenen Meinung auf das wahrgenommene Meinungsklima finden sich sowohl mit Blick auf die derzeitige Beliebtheit als auch mit Blick auf die Wahlerwartungen nur vereinzelt, tragen dann aber substanziell zu den Urteilen bei. 


\section{Inhalt}

1. Einleitung 334

2. Derzeitiges und zukünftiges Meinungsklima 336

3. Quellen der Meinungsklimawahrnehmung 337
a) Soziale Projektion 337

b) Medienberichterstattung: Umfragen und Tenor der Darstellung 339

c) Persönliches soziales Umfeld 341

d) Modellbildung $\quad 342$

4. Anlage der Untersuchung 344

5. Ergebnisse 346

a) Die Wahrnehmung des Meinungsklimas und der relevanten Einflussfaktoren $\quad 346$

b) Einflüsse auf die Wahrnehmung des Meinungsklimas 348

(1) Derzeitiges Meinungsklima (Beliebtheit der Parteien) 348

(2) Zukünftiges Meinungsklima (Wahlerwartungen) 350

Erwartete prozentuale Stimmenanteile $\quad 350$

Erwartungen zum Einzug der Kleinparteien ins Parlament 351

6. Zusammenfassung und Diskussion 354

\section{Einleitung}

Die Vorstellungen, die Wählerinnen und Wähler von den politischen Meinungen und Einstellungen anderer Menschen und ihrem voraussichtlichen Wahlverhalten haben, finden in der Politikwissenschaft zunehmend Beachtung. Im Mittelpunkt stehen dabei in erster Linie die Wahlerwartungen der Bürger, verstanden als ihre Vermutungen darüber, wie eine bevorstehende Wahl ausgehen wird. Das Interesse an Wahlerwartungen ergibt sich nicht zuletzt daraus, dass ihnen ein Einfluss auf das individuelle Wahlverhalten unterstellt wird. In entsprechenden empirischen Untersuchungen werden Wähler in der Regel gebeten, bestimmte Szenarien zu beurteilen (Blais u. a. 2008), oder Vorhersagen bezüglich des Wahlgewinners (Delavande/ Manski 2012), des Zustandekommens bestimmter Koalitionen (Huber u. a. 2009) oder des Einzugs kleinerer Parteien ins Parlament (z. B. Faas/Schmitt-Beck 2007) zu treffen.

Die bisherige Forschung zu Wahlerwartungen lässt drei Schwerpunkte erkennen: Erstens interessiert man sich für ihre Ursachen, also die Frage, welche Informationen bzw. Quellen der Einzelne heranzieht, um zu entsprechenden Einschätzungen 
zu kommen (z. B. Blais/Bodet 2006; Delavande/Manski 2012; Faas/Schmitt-Beck 2007; Irwin/van Holsteyn 2002; Krizan/Miller/Johar 2010). Zweitens geht es um die Qualität bzw. die Genauigkeit der Erwartungen (z. B. Huber u. a. 2009; LewisBeck/Skalaban 1989; Lewis-Beck/Tien 1999). Und drittens beschäftigt man sich mit ihren Auswirkungen auf politische Präferenzen und Verhalten, insbesondere die Wahlabsicht (Gimpel/Harvey 1997; Morwitz/Pluzinski 1996), die Wahlentscheidung (Bargsted/Kedar 2009; Huber u. a. 2009) und die Wahlbeteiligung (Hoffmann/ Klein 2013). Der vorliegende Beitrag ist der ersten Gruppe zuzuordnen und befasst sich mit den Ursachen parteibezogener Wahlerwartungen im Vorfeld der Bundestagswahl 2013. Dazu erweitern wir die bisherigen Betrachtungen in der Literatur um zwei wesentliche Aspekte.

Erstens konzentriert sich, von einigen Ausnahmen abgesehen, die Mehrzahl der bisherigen einschlägigen Untersuchungen zur Entstehung von Wahlerwartungen bzw. Meinungsklimawahrnehmungen auf einzelne mögliche Einflussquellen und hier vor allem auf Umfragen. In Anlehnung an neuere Untersuchungen (Blais/Bodet 2006; Huber u. a. 2009), gehen wir dagegen davon aus, dass Umfragen nur eine von mehreren Informationsquellen sind, die zur Ausbildung von Wahlerwartungen beitragen. Neben der gut belegten Tendenz, die eigenen politischen Einstellungen auf andere Menschen zu projizieren (z. B. Granberg/Brent 1983; Krizan/Miller/Johar 2010; Marks/Miller 1987; Meffert u. a. 2011), spielen als weitere Wahrnehmungsquellen auch Meinungsverteilungen im persönlichen Umfeld (O'Gorman 1979; Uhlaner/Grofman 1986) sowie die Wahrnehmung des generellen Tenors der Medienberichterstattung eine Rolle (Gunther 1998). Zwar liegen zu fast allen genannten Einflüssen bereits isoliert empirische Belege vor, eine ganzheitliche Betrachtung ihrer relativen Einflussstärke steht bislang allerdings noch aus.

Zweitens differenzieren wir die Vorstellungen, die der Einzelne von Meinungen und Verhaltensweisen anderer Menschen hat, in zeitlicher Hinsicht und betrachten nicht nur Wahlerwartungen als in die Zukunft gerichtete Urteile, sondern auch die Wahrnehmung gegenwärtiger Meinungsverteilungen in der Bevölkerung und zwar im Hinblick auf die aktuelle Beliebtheit der Parteien. Beide Urteile kann man als Wahrnehmungen des Meinungsklimas betrachten, einmal des derzeitigen (Beliebtheit der Parteien) und einmal des zukünftigen (Wahlerwartungen).

Wir interessieren uns im Folgenden für die relative Einflussstärke der genannten Quellen auf die Wahrnehmungen des politischen Meinungsklimas. Dazu haben wir im Vorfeld der Bundestagswahl 2013 eine repräsentative Befragung unter 1.012 Berliner Bürgern durchgeführt. Neben einer Einschätzung zur derzeitigen Beliebtheit der Parteien in Deutschland, als Ausdruck des aktuellen Meinungsklimas, haben wir diese außerdem nach ihren Erwartungen zum voraussichtlichen Abschneiden 
der Parteien bei der Bundestagswahl gefragt. Für beide Urteile beziehen wir die konkrete Erinnerung an aktuelle Umfrageergebnisse, den generellen Medientenor, die persönlichen Einstellungen sowie die im sozialen Umfeld wahrgenommenen Meinungen zu den einzelnen Parteien als mögliche Ursachen in die Analyse ein.

\section{Derzeitiges und zukünftiges Meinungsklima}

Im Hinblick auf die Einschätzung der politischen Meinungen oder Verhaltensweisen Anderer kann man grundsätzlich zwei Perspektiven unterscheiden. Zum einen kann man die Wahrnehmung des derzeitigen Meinungsklimas betrachten, beispielsweise die Beurteilung der aktuellen Stärke oder Beliebtheit einer Partei, einer Person oder thematischen Position in der Bevölkerung. Man kann aber auch nach subjektiven Prognosen fragen, also der voraussichtlichen Meinung der Bevölkerung zu einem bestimmten Zeitpunkt in der Zukunft. Zur letzteren Kategorie zählen auch Erwartungen an den Wahlausgang (Shamir/Shamir 2000: 121).

Die Trennung zwischen derzeitigem und zukünftigem Meinungsklima wurde erstmals durch Noelle-Neumann im Rahmen der Theorie der Schweigespirale vorgenommen, wofür sie zwei Begründungen anführt: Erstens geht sie davon aus, dass die Wahrnehmung des zukünftigen Meinungsklimas stärkere Verhaltenskonsequenzen nach sich zieht als Einschätzungen des Status quo: „If there is a divergence in the assessment of the present and future strengths of a particular view, it is the expectation of the future position which will determine the extent to which the individual is willing to expose himself." (Noelle-Neumann 1974: 45). Zweitens sind für sie Divergenzen zwischen beiden Urteilen Zeichen bestehender Wandlungsprozesse innerhalb der öffentlichen Meinung und daher Ausdruck ihrer Dynamik. Hohe Korrelationen zwischen derzeitiger und zukünftiger Meinungsklimawahrnehmung deuten hingegen auf einen eher stabilen Zustand der öffentlichen Meinung hin (Noelle-Neumann 1974). Einschätzungen über das derzeitige und zukünftige Meinungsklima können also differieren, wenngleich sich empirisch durchaus starke Zusammenhänge zeigen (Marsh 1985; Petric/Pinter 2002; Shamir/Shamir 2000: 133).

Theoretische Ansätze sehen dabei in der Regel die Einschätzung des zukünftigen Meinungsklimas als der gegenwärtigen Wahrnehmung nachgelagertes Urteil (Petric/Pinter 2002; Taylor 1982), einige siedeln beide Einschätzungen allerdings auch auf der gleichen Ebene an (z. B. Schenk 2007: 530). Wir folgen der ersten Konzeption und nehmen mit Blick auf die politischen Parteien einen Zusammenhang zwischen der Wahrnehmung ihrer derzeitigen Beliebtheit und den Erwartungen hinsichtlich ihres Abschneidens bei der Wahl an. Dabei dient die Einschätzung der 
aktuellen Stärke der Partei als Basis für die jeweilige Wahlerwartung. Daraus folgt unsere Hypothese 1:

H1: Je höher die derzeitige Beliebtheit einer Partei eingeschätzt wird, desto positiver fallen die Erwartungen hinsichtlich des Abschneidens der Partei bei der Bundestagswahl aus.

Für eine getrennte Betrachtung der Urteile zum derzeitigen und zukünftigen Meinungsklima spricht außerdem, dass einzelne Quellen, aus denen sich beide Einschätzungen speisen, möglicherweise mit jeweils unterschiedlichem Gewicht eingehen. Beispielsweise können Shamir und Shamir (2000) in einer qualitativen Studie zeigen, dass Erwartungen an zukünftige Meinungsverteilungen in stärkerem Maße auf der Bewertung aktueller politischer, wirtschaftlicher und sozialer Rahmenbedingungen bzw. Ereignisse basieren als dies bei Einschätzungen des derzeitigen Meinungsklimas der Fall ist. Gleichzeitig ist auch die Projektion eigener Einstellungen auf das Meinungsklima bei der Erwartungsbildung stärker ausgeprägt (Shamir 1995). Auch bezüglich des medialen Einflusses auf die Meinungsklimawahrnehmung kann man Unterschiede zwischen beiden Urteilen annehmen. So geht Gunther im Rahmen seines „Persuasive Press Inference“-Ansatzes davon aus, dass Menschen starke Medienwirkungen auf andere Menschen annehmen. Diese Annahme hat wiederum zur Folge, dass sie den aktuell wahrgenommenen Medientenor als Meinungsklimaindikator ansehen. Allerdings äußert sich der mediale Einfluss auf die Meinungen anderer Menschen in den Augen der Rezipienten nicht unmittelbar, sondern erst mit zeitlicher Verzögerung. Man kann daher theoretisch von stärkeren Einflüssen des wahrgenommenen Medientenors auf das zukünftig wahrgenommene Meinungsklima ausgehen (Gunther 1998; Gunther/Christen 1999; siehe dazu auch Kapitel 3 b).

Eine quantitative Untersuchung der relativen Bedeutung verschiedener Informationsquellen für die Ausbildung der Vorstellungen von gegenwärtigen und zukünftigen Meinungsverteilungen existiert bislang allerdings nicht. Im folgenden Kapitel gehen wir daher zunächst auf die bedeutsamsten Informationsquellen von Meinungsklimaurteilen ein.

\section{Quellen der Meinungsklimawahrnehmung}

\section{a) Soziale Projektion}

Eines der stabilsten sozialpsychologischen Phänomene ist die menschliche Tendenz zu sozialer Projektion, das heißt der Annahme, die eigenen Einstellungen, Eigenschaften und Verhaltensweisen fänden sich auch bei anderen Menschen (Marks/ Miller 1987). In empirischen Studien kann man dementsprechend häufig Korrela- 
tionen zwischen beiden Urteilen beobachten (Mullen u. a. 1985). Gleichzeitig ist man sich allerdings über die Ursachen von Projektionseffekten weitgehend uneinig (Christen/Gunther 2003; Marks/Miller 1987).

Einflüsse der eigenen Meinung auf das gegenwärtig wahrgenommene Meinungsklima werden in der Forschung meist unter dem Titel „Looking Glass-Effekt“ (Fields/Schuman 1976) bzw. „False Consensus-Effekt“ (Marks/Miller 1987; Ross/ Greene/House 1977; Wojcieszak/Price 2009) behandelt. Auch hier zeigen sich regelmäßig Projektionstendenzen, indem die eigene Meinung tendenziell auch anderen Menschen zugeschrieben wird (Mullen u. a. 1985), wodurch es auch zu verzerrten bzw. ungenauen Vorstellungen bezüglich des herrschenden Meinungsklimas kommen kann (O'Gorman 1979). Der Effekt tritt im Hinblick auf verschiedenste Themen auf (Wojcieszak/Price 2009). Untersuchungen im Bereich der politischen Kommunikation weisen zudem darauf hin, dass die Tendenz zu sozialer Projektion durch ein heterogenes soziales Umfeld abgeschwächt wird, insbesondere wenn man dort auf Gegenmeinungen trifft (Uhlaner/Grofman 1986; Wojcieszak/ Price 2009).

Einflüsse der eigenen Meinung auf die Wahrnehmung des zukünftigen Meinungsklimas werden im Zusammenhang mit Wahlerwartungen auch mit dem Begriff des „Wishful Thinking“ (Krizan/Windschitl 2007) bezeichnet. Damit ist gemeint, dass Wähler mit einer positiven Einstellung zu einer Partei oder einem Kandidaten eher glauben, dass dieser bei einer bevorstehenden Wahl erfolgreich sein wird (Krizan/Miller/Johar 2010; Meffert u. a. 2011; Miller u. a. 2012). Diese Tendenz lässt sich auch dann feststellen, wenn andere „objektive“ Informationen zur Ausbildung einer Wahlerwartung vorliegen, wie etwa Umfrageergebnisse (Delavande/Manski 2012). Außerdem lässt sich zeigen, dass in die Zukunft gerichtete Projektionseffekte stärker sein können als solche auf die Wahrnehmung des derzeitigen Meinungsklimas (Shamir 1995).

Insgesamt können wir auf Basis der bisherigen Forschung davon ausgehen, dass persönliche Meinungen über die deutschen Parteien positiv mit der Wahrnehmung ihrer derzeitigen Beliebtheit sowie den Erwartungen an ihr Abschneiden bei der Bundestagswahl zusammenhängen. Daraus folgen die Hypothesen $2 \mathrm{a}$ und $2 \mathrm{~b}$ :

$\mathrm{H} 2 \mathrm{a}$ : Je positiver die persönliche Meinung zu einer Partei ist, desto höher wird ihre derzeitige Beliebtheit in der Bevölkerung eingeschätzt.

$\mathrm{H} 2 \mathrm{~b}$ : Je positiver die persönliche Meinung zu einer Partei ist, desto positiver fallen die Erwartungen hinsichtlich ihres Abschneidens bei der Bundestagswahl aus. 
Die Wahrnehmung des Meinungsklimas im Bundestagswahlkampf 2013

\section{b) Medienberichterstattung: Umfragen und Tenor der Darstellung}

Dass Medieninhalte individuelle Vorstellungen von Meinungsverteilungen in der Gesellschaft prägen, wird seit längerem vermutet (Katz 1982; Noelle-Neumann 1974) und konnte bereits mehrfach empirisch bestätigt werden. ${ }^{1}$ Einige Autoren kommen sogar zu dem Schluss, dass die Medien wesentlich erfolgreicher darin sind, Vorstellungen vom Denken und Handeln Anderer zu prägen, als unmittelbar persuasiv auf die eigenen Einstellungen bzw. das eigene Verhalten zu wirken (Mutz 1998: 5; Tsfati 2003: 66). Obwohl bezüglich des Einflusses der Medien auf das wahrgenommene Meinungsklima weitgehend Einigkeit besteht, bleibt die Forschung zur Frage, welche medialen Inhalte genau zu diesen Vorstellungen führen, bislang weitgehend fragmentiert. Bei näherer Betrachtung lassen sich allerdings zwei Kategorien medial vermittelter Meinungsklimahinweise (,cues“) ausmachen.

Explizite Hinweise beschreiben das herrschende Meinungsklima direkt und in aggregierter Form. Am bedeutsamsten sind dabei Umfragen, die - insbesondere im Vorfeld von Wahlen - mittlerweile fester Bestandteil der Berichterstattung sind (Brettschneider 2008; o. V. 2013). Aber auch subjektive Aussagen über das Meinungsklima, die sich nicht auf demoskopische Daten stützen (z. B. „Die Deutschen lehnen Steuererhöhungen mehrheitlich ab.“), sind expliziter Natur und können Meinungsklimawahrnehmungen beeinflussen (Donsbach/Weisbach 2005; Mayer 2001), beispielsweise wenn sie von Wahlkampfexperten stammen (Huber 2011). Tatsächlich machen solche subjektiven Hinweise auf das Meinungsklima einen erheblichen Teil der Mediendarstellung des Meinungsklimas aus (Reinemann u. a. 2013: 104). Ein Rezipient, der solche Hinweise wahrnimmt, kann die entsprechenden Informationen zumindest potenziell in seine Einschätzung des Meinungsklimas einfließen lassen. ${ }^{2}$ Gerade Umfragen kommt dabei besondere Bedeutung zu: Ihre Anzahl hat sich in den vergangenen deutschen Bundestagswahlkämpfen deutlich erhöht (Brettschneider 2008) und auch die Bürger nehmen sie regelmäßig wahr (Faas/Schmitt-Beck 2007; Partheymüller/Schäfer 2013: 582). Zudem deuten aktuelle Studien darauf hin, dass Umfragen eine zentrale Rolle für die Wahrnehmung des derzeitigen Meinungsklimas wie auch für die Entstehung von Wahlerwartungen spielen (Blais/Bodet 2006; Irwin/van Holsteyn 2002; Meffert u. a. 2011). Insbesondere politisch involvierte Bürger und solche, die dem Wahlkampf insgesamt sowie der Medienberichterstattung große Aufmerksamkeit schenken, lassen sich in

1 Siehe zum Beispiel Brosius/Bathelt 1994; Daschmann 2001; Gunther 1998; Mutz 1997; Mutz/Soss 1997; Tsfati/Stroud/Chotiner 2013.

$2 \mathrm{Ob}$ dies tatsächlich geschieht, hängt natürlich von zahlreichen weiteren Faktoren ab, wie etwa der Glaubwürdigkeit der Quelle oder der kognitiven Kapazität des Rezipienten. 
ihren Wahlerwartungen von Umfrageergebnissen beeinflussen, und zwar umso stärker, je näher der Wahltermin rückt (Krizan/Sweeny 2013). Dabei sind Effekte auf die Koalitionserwartungen ebenso beobachtbar wie solche auf die Einschätzung, ob kleinere Parteien den Einzug in den Bundestag schaffen (Faas/Schmitt-Beck 2007) bzw. wie gut eine Partei voraussichtlich insgesamt abschneiden wird (Irwin/ van Holsteyn 2002). Die regelmäßige Rezeption von Umfrageergebnissen führt außerdem dazu, dass die Wahlerwartungen genauer werden (Meffert u. a. 2011). In der vorliegenden Untersuchung berücksichtigen wir als ersten medialen Einflussfaktor auf die Meinungsklimawahrnehmung die durch die Bürger wahrgenommenen Umfrageergebnisse:

H3 a: Je höher der mit Blick auf eine Partei erinnerte Umfragewert, desto höher wird ihre derzeitige Beliebtheit in der Bevölkerung eingeschätzt.

H3 b: Je höher der mit Blick auf eine Partei erinnerte Umfragewert, desto positiver fallen die Erwartungen hinsichtlich ihres Abschneidens bei der Bundestagswahl aus.

Implizite Hinweise sind Medieninhalte, die nicht direkt in Meinungsklimaurteile übersetzt werden können, sondern einer weitergehenden kognitiven Verarbeitung bedürfen, die in der Regel heuristischer Natur ist. Dazu zählen vor allem wertende Inhalte, wie zum Beispiel Fallbeispiele, als exemplarisch in den Medien präsentierte Einzelmeinungen (Brosius/Bathelt 1994; Daschmann 2001; Zillmann 2002), aber auch der generelle Tenor der Berichterstattung (Gunther 1998; Gunther/Christen 1999; Gunther u. a. 2001). Die Fallbeispielforschung geht davon aus, dass Menschen medial präsentierte Einzelfälle auf Basis weniger Eigenschaften verallgemeinern und so eine Vorstellung von größeren Gruppen von Menschen ausbilden (Repräsentativitätsheuristik) (Kahneman/Tversky 1973; Zillmann 2006). In Bezug auf Meinungsklimaeinschätzungen dominieren diese exemplarisch präsentierten Informationen in vielen Fällen sogar den Einfluss summarischer Realitätsbeschreibungen, beispielsweise in Form von Umfragen (base-rate fallacy) (Zillmann 2002: 27).

Wirkungen des Tenors der Berichterstattung werden ebenfalls heuristisch erklärt, indem man annimmt, dass Rezipienten den Massenmedien starke Wirkungen auf andere Menschen zuschreiben (Davison 1983; Gunther/Storey 2003). Dementsprechend glauben sie auch an persuasive Effekte tendenziöser Berichterstattung. Weiterhin wird unterstellt, dass Rezipienten von einer hohen Reichweite des medialen Angebots ausgehen und glauben, andere Menschen seien mit ähnlichen Inhalten konfrontiert. Auf Basis dieser Annahmen zur Nutzung und Wirkung von Medieninhalten verallgemeinern sie den vermuteten Effekt einer tendenziösen Berichterstattung und schließen so auf entsprechende Meinungsverteilungen in der Gesellschaft. Vereinfacht gesagt wird in den Augen der Rezipienten das, was heute be- 
richtet wird, morgen zur Meinung vieler. Diese als „Persuasive Press Inference“ (PPI) bekannte Heuristik (Gunther 1998) konnte ebenfalls bereits in mehreren Studien nachgewiesen werden (ebd.; Gunther/Christen 2002). Weitergehende Untersuchungen konnten außerdem zeigen, dass Eindrücke vom herrschenden Meinungsklima in erster Linie dem wahrgenommenen und weniger dem tatsächlichen Medientenor folgen. Dies ist deshalb wichtig, da die Wahrnehmung der gleichen Medieninhalte, je nach eigener Einstellung, erheblich differieren kann (Christen/Kannaovakun/Gunther 2002; Vallone/Ross/Lepper 1985). Entsprechend ergeben sich für die Effekte der Wahrnehmung des generellen Medientenors gegenüber den Parteien folgende Annahmen:

H4 a: Je positiver der Tenor der Berichterstattung über eine Partei wahrgenommen wird, desto höher wird die derzeitige Beliebtheit der Partei in der Bevölkerung eingeschätzt.

H4 b: Je positiver der Tenor der Berichterstattung über eine Partei wahrgenommen wird, desto positiver fallen die Erwartungen hinsichtlich des Abschneidens der Partei bei der Bundestagswahl aus.

\section{c) Persönliches soziales Umfeld}

Neben den Medien wird auch dem sozialen Umfeld eine zentrale Rolle bei der Vermittlung von Meinungsklimaeindrücken zugeschrieben. Einen frühen Hinweis darauf gibt Davison (1958: 99 f.), der beschreibt, wie Menschen sich Meinungen bei anderen Personen gezielt oder unabsichtlich einholen, um eine Vorstellung von der Verteilung bestehender Ansichten zu erhalten. Was er als ,person sampling“ bezeichnet, entspricht im Grunde der Rolle, die das persönliche Umfeld auch in der Theorie der Schweigespirale einnimmt (Noelle-Neumann 1974) und andere Autoren ebenfalls andeuten (Wojcieszak/Price 2009: 29). Demnach empfängt das Individuum verschiedenste Signale aus seiner Umgebung und interpretiert sie mit Blick auf das herrschende Meinungsklima. Dazu gehören neben öffentlich gezeigten Verhaltensweisen, wie etwa dem Tragen von Zeichen, Aufklebern, Demonstrationen, Buh- oder Beifallsrufen auch öffentlich artikulierte Meinungen die dem Einzelnen Hinweise auf das Meinungsklima geben (Noelle-Neumann 1974, 2001; NoelleNeumann/Petersen 2004; Shamir 1995: 26).

Bislang existieren nur wenige Studien, die den Einfluss des sozialen Umfelds auf das wahrgenommene Meinungsklima untersuchen (z. B. O'Gorman 1979; Wojcieszak/Price 2009). Empirisch belegt ist aber eine relativ große Übereinstimmung zwischen der eigenen Meinung und der des engeren persönlichen Netzwerks (z. B. Familie), die maßgeblich durch eine generell ausgeprägte Homogenität des engeren 
Umfelds bedingt ist (Mutz 1995: 46 ff.; Schenk 1995). Die Wahrscheinlichkeit auf andere Meinungen zu stoßen steigt mit der Heterogenität des persönlichen Netzwerks, die ihrerseits mit der sozialen Distanz zum engeren Umfeld wächst (z. B. am Arbeitsplatz oder im Verein) (Mutz 2006: 25 ff.). Solche gegenläufigen Meinungen beeinflussen auch den Eindruck vom herrschenden Meinungsklima, indem sie Tendenzen zur Projektion der eigenen Meinung auf Andere abschwächen (Wojcieszak/ Price 2009), und scheinen zudem die Genauigkeit der Urteile zu verbessern (O'Gorman 1979).

Auch mit Blick auf die Wahlerwartungen ist ein Effekt des persönlichen Umfelds zu vermuten. Darauf deuten Untersuchungen hin, die Einflüsse des Wohnorts (z. B. einer Region, Stadt oder eines Wahlbezirks) auf die Einschätzungen zum Wahlausgang feststellen (Babad/Hills/O'Driscoll 1992; Blais/Bodet 2006; Meffert u. a. 2011). Babad, Hills und O’Driscoll (1992) sprechen hier auch vom „Neighborhood Effect". Allerdings betrachten bisherige Studien vergleichsweise große geografische Regionen (z. B. Ost- und Westdeutschland oder einzelne Städte) und lassen daher kaum Rückschlüsse auf die Effekte des engeren persönlichen Umfelds zu. Des Weiteren dürfte für das Zustandekommen individueller Wahlerwartungen ähnlich wie bei den medienvermittelten Hinweisen - weniger die tatsächliche Meinungsverteilung im persönlichen Umfeld relevant sein, sondern die wahrgenommene. Aus diesem Grund betrachten wir in der vorliegenden Studie Letzteres ebenfalls als mögliche Ursache der Meinungsklimawahrnehmung.

H5 a: Je positiver die im persönlichen Umfeld wahrgenommene Meinung zu einer Partei ist, desto höher wird die derzeitige Beliebtheit der Partei in der Bevölkerung eingeschätzt.

H5 b: Je positiver die im persönlichen Umfeld wahrgenommene Meinung zu einer Partei ist, desto positiver fallen die Erwartungen hinsichtlich des Abschneidens der Partei bei der Bundestagswahl aus.

\section{d) Modellbildung}

Die bisherigen Überlegungen verdeutlichen drei Aspekte: (1) Die Betrachtung der verschiedenen Quellen der Meinungsklimawahrnehmung sollte nicht getrennt, sondern integrativ erfolgen, da die einzelnen Faktoren sich gegenseitig beeinflussen. Das gilt insbesondere für soziale Projektionseffekte, die durch externe Informationen aus den Massenmedien oder dem sozialen Umfeld abgeschwächt werden können. Zudem können die schwierig zu isolierenden Effekte von Informationsquellen mit hoher Meinungskonsonanz (insbesondere das persönliche Umfeld und die persönliche Meinung) besser bestimmt werden. (2) Für die Wahrnehmung des Mei- 
nungsklimas ist zudem weniger entscheidend, wie sich die Meinungsverteilung im sozialen Umfeld, der Tenor der Berichterstattung oder die Umfrageberichterstattung tatsächlich darstellen, sondern wie sie wahrgenommen werden. (3) Aktuelle Meinungsklimaurteile und solche, die sich auf das zukünftige Meinungsklima beziehen (z. B. Wahlerwartungen), müssen getrennt betrachtet werden, da jeweils Unterschiede in der Bedeutung einzelner Wahrnehmungsquellen zu erwarten sind.

Auf Basis dieser Überlegungen haben wir ein Pfadmodell der Meinungsklimawahrnehmung und Wahlerwartungen in Bezug auf politische Parteien abgeleitet, in das die eigene Meinung zu einer Partei, die im sozialen Umfeld wahrgenommene Meinung, die wahrgenommene Tendenz der Berichterstattung über die Partei sowie die zur Partei erinnerten Umfragewerte als exogene Variablen eingehen (Abbildung 1). Das Modell erlaubt (1) die relative Einflussstärke der genannten Einflussfaktoren kontrolliert zu bestimmen und zwar (2) jeweils getrennt nach der Wahrnehmung des derzeitigen und zukünftigen Meinungsklimas als endogene Variablen. Es ermöglicht darüber hinaus (3) die Analyse indirekter Effekte, die die vier exogenen Variablen vermittelt über die Wahrnehmung des derzeitigen Meinungsklimas auf die Wahlerwartungen ausüben können. Vergleiche über verschiedene Parteien hinweg erlauben (4) die Identifikation genereller und spezifischer Muster in den Urteilsstrukturen der Bürger.

Abbildung 1: Quellen der Wahrnehmung des derzeitigen Meinungsklimas (Beliebtheit) und des künftigen Meinungsklimas (Wahlerwartungen)

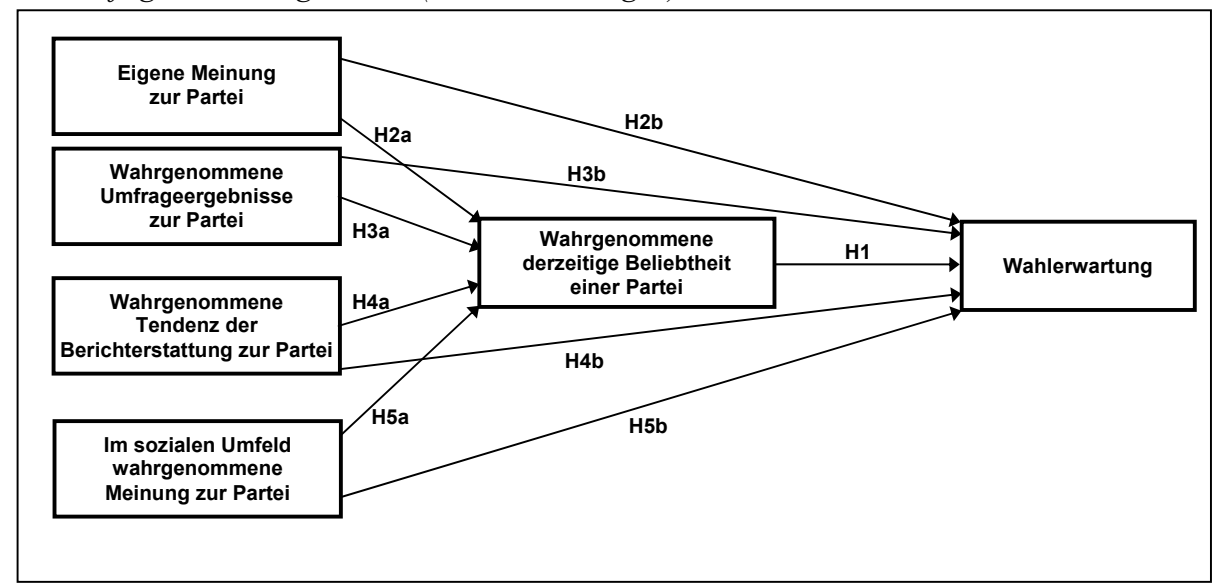




\section{Anlage der Untersuchung}

Um unsere Hypothesen zu prüfen, haben wir im Vorfeld der Bundestagswahl 2013 eine repräsentative Befragung unter 1.012 Berliner Bürgern durchgeführt. Wir haben uns für eine regional begrenzte Medien- und Bevölkerungsstichprobe in Berlin entschieden, um für einen noch ausstehenden Teil der Studie die von den Wählern genutzten Medieninhalte möglichst umfassend als mögliche Einflussfaktoren auf ihre Wahrnehmungen und ihr Wahlverhalten einbeziehen zu können (ausführlich Reinemann u. a. 2013). Weil sich viele Wähler insbesondere auch aus regionalen Tageszeitungen informieren, müsste man bei einer bundesweiten Stichprobe eine kaum zu bewältigende Menge an Medien betrachten, würde aber andererseits den theoretischen Wert der Erklärungen nicht steigern. Da die Ergebnisse der Inhaltsanalyse noch nicht vorliegen, können wir sie im vorliegenden Beitrag allerdings noch nicht berücksichtigen. Für Berlin sprachen außerdem die politisch vielfältige Medienlandschaft und die relativ hohe Zustimmung zu verschiedenen Kleinparteien. All diese Merkmale ermöglichen trotz der regionalen Begrenztheit differenzierte Analysen unterschiedlicher Wählersegmente.

Die telefonische Befragung wurde vom sozialwissenschaftlichen Umfragezentrum der Universität Duisburg-Essen durchgeführt. Sie fand vom 9.9.2013 bis 14.9.2013 statt, also zwei Wochen vor der Bundestagswahl und in der Woche unmittelbar vor der Landtagswahl in Bayern. ${ }^{3}$ Im Zuge der Befragung sollten die Teilnehmer Auskunft zu den in unserem untersuchungsleitenden Modell dargestellten, für Meinungsklimawahrnehmungen entscheidenden Merkmalsbereichen geben (siehe Abbildung 1). Dies waren im Einzelnen:

- Eigene Meinung zu den Parteien. Die persönliche Meinung zu den sieben wichtigsten Parteien im Bundestagswahlkampf 2013 (CDU/CSU, SPD, Bündnis 90/ Die Grünen, FDP, Die Linke, Piratenpartei und AfD) wurde über folgendes Item abgefragt: ,Jetzt interessiert uns Ihre Meinung von den Parteien. Bitte sagen Sie

3 Grundgesamtheit waren die in Privathaushalten mit Telefonanschluss lebenden, bei der Bundestagswahl wahlberechtigten Bürgerinnen und Bürger Berlins. Zur Auswahl der Haushalte wurde eine Zufallsstichprobe nach Gabler/Häder (1997) realisiert. Die Auswahl der Zielpersonen im Haushalt erfolgte nach der Last-Birthday-Methode, die Zahl der Kontaktversuche lag bei 10. Der Rücklauf bewegte sich mit 20 Prozent in einem für Telefonumfragen typischen Bereich. 52 Prozent der Befragten waren männlich, das Durchschnittsalter der Befragten lag bei knapp 55 Jahren $(S D=17,6)$, 50 Prozent hatten die Hochschulreife (Gymnasium/EOS), 84 Prozent war der Ausgang der Bundestagswahl ,wichtig“ oder „sehr wichtig“ und 63 Prozent hatten eine längerfristige Parteibindung. Damit ist die Stichprobe wie bei thematisch ähnlichen Befragungen männlicher, älter, besser gebildet und politisch interessierter als die Gesamtbevölkerung und entspricht in ihrer Struktur eher den tatsächlichen Wählern. Auf eine Gewichtung wurde verzichtet, da es im vorliegenden Beitrag vorwiegend um Zusammenhänge zwischen Konstrukten und nicht um Anteilsschätzungen geht. 
uns, ob Sie von den einzelnen Parteien alles in allem eine gute oder eine schlechte Meinung haben. Wie ist das bei [Partei]?“. Auf einer fünfstufigen Likert-Skala von 1 für „sehr schlechte Meinung“ bis 5 ,sehr gute Meinung“ konnten die Befragten ihre Urteile abgeben.

- Meinungen über die Parteien im persönlichen Umfeld. Hier sollten die Befragten einschätzen, welche Meinung ihre Familie und Freunde zu den einzelnen Parteien haben (,Wir haben ja vorhin über ihre eigene Meinung, die Medien und die Deutschen insgesamt gesprochen. Jetzt interessiert uns noch, wie es in Ihrem persönlichen Umfeld aussieht, also in ihrer Familie und bei Ihren Freunden: Welche Meinung hat ihr Umfeld von den einzelnen Parteien?"). Die Urteile konnten für jede der sieben Parteien auf der fünfstufigen Likert-Skala abgegeben werden, die auch für die eigene Meinung verwendet wurde.

- Wahrgenommener Medientenor. Analog wurde ebenfalls auf einer fünfstufigen Skala die Wahrnehmung des generellen Medientenors gegenüber den einzelnen Parteien abgefragt. Die Frage lautete: „Und wie haben die Medien Ihrer Meinung nach in der vergangenen Woche allgemein über die einzelnen Parteien berichtet? Ich nennen Ihnen jetzt noch einmal die Parteien. Wenn Sie Ihre Meinung auf einer Skala von positiv bis negativ einstufen: Wie positiv bzw. negativ war im Allgemeinen die Berichterstattung über [Partei]?"“. Ein Wert von 1 stand für einen sehr negativen, ein Wert von 5 für einen sehr positiven Medientenor.

- Umfrageerinnerung. Zunächst wurden die Befragten gebeten anzugeben, mit welcher Häufigkeit sie Meinungsumfragen in den Medien wahrgenommen haben. Bei denjenigen, die Umfragen wahrgenommen hatten, wurde zudem die Erinnerung an konkrete Umfrageergebnisse mithilfe einer offenen Angabe erfasst (,Wissen Sie vielleicht auch noch, welche Prozentanteile die einzelnen Parteien ungefähr in den Umfragen hatten, die Sie zuletzt gesehen haben? Wie war das mit [Partei]?“).

- Derzeitiges Meinungsklima (Beliebtheit). Die Einschätzung des derzeitigen Meinungsklimas haben wir anhand der momentanen Beliebtheit der Parteien erfasst (,Jetzt geht es noch einmal um die Beliebtheit der Parteien. Diesmal würden wir aber gerne von Ihnen wissen, wie die Deutschen Ihrer Einschätzung nach allgemein über die Parteien denken. Dazu lese ich Ihnen die Parteien noch einmal vor. Bitte stufen Sie Ihr Urteil auf einer Skala von 1: sehr schlechte Meinung bis 5: sehr gute Meinung ab. Welche Meinung haben die Deutschen von [Partei]?“).

- Künftiges Meinungsklima (Wahlerwartungen). Die Prognose des zukünftigen Meinungsklimas in Form der Wahlerwartung wurde über zwei Items erfasst. Zunächst sollten die Befragten schätzen, welchen Zweitstimmenanteil die Parteien bei der bevorstehenden Bundestagswahl erreichen würden (,Mal abgesehen von 
der derzeitigen Stimmung. Was glauben Sie: Wie viel Prozent der Stimmen werden am 22. September bei der Bundestagswahl auf die einzelnen Parteien entfallen? Wie viel Prozent bekommt ...?“). Darüber hinaus ging es um den Einzug der Kleinparteien (,Nicht alle antretenden Parteien werden ja nach der Wahl in den Bundestag einziehen. Manche werden die 5\%-Hürde nicht schaffen. Ich nenne Ihnen jetzt einige Parteien und Sie sagen mir bitte jeweils, ob Sie glauben, dass die Partei die 5\%-Hürde deutlich überspringt, knapp überspringt, genau bei $5 \%$ landet, sie knapp verfehlt oder deutlich verfehlt. Wie ist das mit [Partei]?”).

\section{Ergebnisse}

\section{a) Die Wahrnehmung des Meinungsklimas und der relevanten Einflussfakto- ren}

Die deskriptive Analyse der hier relevanten Wahrnehmungen und Urteile vermittelt ein anschauliches Bild des Meinungsklimas, das im Vorfeld der Bundestagswahl 2013 unter unseren Befragten herrschte: So beurteilten die Berliner Wählerinnen und Wähler SPD, Union und Bündnis 90/Die Grünen am besten, Piraten, FDP und AfD am schlechtesten. Die Linke lag in den persönlichen Meinungen etwa in der Mitte zwischen diesen beiden Gruppen. Die Wahrnehmung der Meinungen im persönlichen Umfeld war den eigenen Meinungen zumindest im Aggregat sehr ähnlich. Deutlich abweichend ist dagegen das Muster, welches sich für die Wahrnehmung des Medientenors ergibt. So hatten unsere Befragten den Eindruck, dass die Medien die Union mit Abstand am positivsten darstellten. Überraschend ist auch die Wahrnehmung des Medientenors bezüglich der FDP: Unsere Befragten nahmen den Medientenor zur FDP nicht nur als sehr viel positiver wahr als ihre eigenen Meinungen und die in ihrem Umfeld, auch das Verhältnis zu den anderen Parteien war nun ein anderes. Anders als in den persönlichen Meinungen und im sozialen Umfeld lag die FDP in den Augen der Befragten in der Medienberichterstattung eher auf einem Niveau mit Grünen und Linken, während Piraten und AfD aus ihrer Sicht sehr viel negativer bewertet wurden (Tabelle 1).

Die erinnerten und erwarteten Wahlergebnisse benötigen eigentlich eine eigenständige Betrachtung, die aber hier nicht geleistet werden kann, weil andere Aspekte im Vordergrund stehen. Es sei deshalb an dieser Stelle nur bemerkt, dass diejenigen, die sich an Umfragen erinnerten, im Mittel durchaus realistische Umfragewerte nannten. Jedoch muss man dabei berücksichtigen, dass es einerseits viele Befragte gab, die sich gar nicht an konkrete Umfragezahlen erinnern konnten. Ihr Anteil schwankte und lag für die CDU und die SPD bei etwa 33 Prozent, für die Grünen bei 39, für die FDP bei 42, für die Linke bei 47, für die Piraten bei 59 und im Falle 
der AfD sogar bei 63 Prozent. ${ }^{4}$ Andererseits ist eine erhebliche Varianz der Schätzungen zu beobachten, was auch an den Standardabweichungen deutlich wird. Bemerkenswert ist außerdem, dass die erwarteten Wahlergebnisse unterschiedlich stark von den erinnerten Umfragewerten abweichen. So nahmen die Befragten beispielsweise an, dass die FDP die Fünf-Prozent-Hürde deutlicher überspringen würde, als es die von ihnen erinnerten Umfragen suggerierten. Diese optimistische Betrachtung der Chancen der FDP spiegelt sich auch in der Frage nach dem Einzug

Tabelle 1: Deskriptive Statistiken (Mittelwerte und Standardabweichungen)

\begin{tabular}{|c|c|c|c|c|c|c|c|}
\hline & Union & SPD & Grüne & FDP & Linke & Piraten & AfD \\
\hline $\begin{array}{l}\text { Eigene Meinung } \\
(n=748 \text {-998) }\end{array}$ & $\begin{array}{c}2,78 \\
(1,06)\end{array}$ & $\begin{array}{l}2,96 \\
(0,94)\end{array}$ & $\begin{array}{l}2,75 \\
(1,13)\end{array}$ & $\begin{array}{c}1,79 \\
(1,06)\end{array}$ & $\begin{array}{c}2,36 \\
(1,22)\end{array}$ & $\begin{array}{c}1,84 \\
(1,04)\end{array}$ & $\begin{array}{c}1,63 \\
(1,02)\end{array}$ \\
\hline $\begin{array}{l}\text { Meinung } \\
\text { persönliches Umfeld } \\
(n=710-875)\end{array}$ & $\begin{array}{c}2,77 \\
(1,14)\end{array}$ & $\begin{array}{c}3,15 \\
(0,88)\end{array}$ & $\begin{array}{c}2,92 \\
(1,08)\end{array}$ & $\begin{array}{c}1,81 \\
(0,95)\end{array}$ & $\begin{array}{c}2,35 \\
(1,16)\end{array}$ & $\begin{array}{c}1,82 \\
(1,02)\end{array}$ & $\begin{array}{c}1,60 \\
(0,98)\end{array}$ \\
\hline $\begin{array}{l}\text { Tendenz der } \\
\text { Berichterstattung } \\
(n=647-928)\end{array}$ & $\begin{array}{c}3,56 \\
(0,82)\end{array}$ & $\begin{array}{c}3,19 \\
(0,80)\end{array}$ & $\begin{array}{c}2,86 \\
(0,77)\end{array}$ & $\begin{array}{c}2,61 \\
(0,88)\end{array}$ & $\begin{array}{c}2,47 \\
(0,89)\end{array}$ & $\begin{array}{c}1,94 \\
(0,92)\end{array}$ & $\begin{array}{l}2,00 \\
(0,99)\end{array}$ \\
\hline $\begin{array}{l}\text { Erinnerte Umfrageer- } \\
\text { gebnisse in \% } \\
(n=280-680)\end{array}$ & $\begin{array}{l}39,3 \\
(5,3)\end{array}$ & $\begin{array}{l}27,7 \\
(5,4)\end{array}$ & $\begin{array}{l}11,5 \\
(3,6)\end{array}$ & $\begin{array}{c}5,8 \\
(2,8)\end{array}$ & $\begin{array}{c}8,5 \\
(3,6)\end{array}$ & $\begin{array}{c}3,6 \\
(2,1)\end{array}$ & $\begin{array}{c}3,1 \\
(1,8)\end{array}$ \\
\hline $\begin{array}{l}\text { Momentane } \\
\text { Beliebtheit in der Ge- } \\
\text { samtbevölkerung } \\
(n=764-976)\end{array}$ & $\begin{array}{c}3,61 \\
(0,77)\end{array}$ & $\begin{array}{c}3,18 \\
(0,70)\end{array}$ & $\begin{array}{l}2,85 \\
(0,75)\end{array}$ & $\begin{array}{c}2,09 \\
(0,80)\end{array}$ & $\begin{array}{l}2,15 \\
(0,86)\end{array}$ & $\begin{array}{c}1,77 \\
(0,90)\end{array}$ & $\begin{array}{r}1,76 \\
(0,92)\end{array}$ \\
\hline $\begin{array}{l}\text { Erwartetes } \\
\text { Wahlergebnis in \% } \\
(n=822-934)\end{array}$ & $\begin{array}{l}38,6 \\
(6,8)\end{array}$ & $\begin{array}{l}30,4 \\
(7,1)\end{array}$ & $\begin{array}{l}12,7 \\
(6,1)\end{array}$ & $\begin{array}{c}6,7 \\
(5,3)\end{array}$ & $\begin{array}{c}8,9 \\
(6,5)\end{array}$ & $\begin{array}{c}4,2 \\
(4,1)\end{array}$ & $\begin{array}{c}3,5 \\
(4,4)\end{array}$ \\
\hline $\begin{array}{l}\text { Erwarteter Einzug } \\
(n=890-1003)\end{array}$ & - & - & $\begin{array}{c}1,32 \\
(0,75)\end{array}$ & $\begin{array}{c}2,50 \\
(1,02)\end{array}$ & $\begin{array}{c}1,72 \\
(1,00)\end{array}$ & $\begin{array}{c}4,00 \\
(1,09)\end{array}$ & $\begin{array}{c}4,31 \\
(0,98)\end{array}$ \\
\hline
\end{tabular}

4 Dies wirft die Frage auf, welchen Einfluss die verschiedenen Quellen von Meinungsklima-Wahrnehmungen bei denjenigen haben, die sich nicht an konkrete Umfragewerte erinnern können. Entsprechende Analysen zeigen für das derzeitige Meinungsklima, dass die Einflussmuster derjenigen mit Erinnerung im Wesentlichen erhalten bleiben, wobei die Effekte der verbliebenen Prädiktoren etwas stärker werden. Für das künftige Meinungsklima zeigt sich, dass die erinnerten Umfragewerte die Erwartungen an die Zweitstimmenanteile insgesamt homogenisieren, auch wenn sich die Schätzungen derjenigen mit und ohne Umfrageerinnerung im Mittel nicht sehr stark unterscheiden. Die kurzfristige Umfrageerinnerung dient hier also vorwiegend als Stabilisator der geschätzten Stimmenanteile. 
der Kleinparteien in den Bundestag wider. So erwarteten 97 Prozent der Befragten einen Einzug der Grünen, 91 Prozent der Linken und immerhin 78 Prozent glaubten, die FDP würde wieder im neuen Parlament vertreten sein. Den Piraten trauten dies dagegen nur 21 Prozent und der AfD sogar nur 14 Prozent zu. Und dies, obwohl AfD und FDP in den letzten Umfragen vor der Wahl fast gleichauf lagen (Tabelle $1)$.

\section{b) Einflüsse auf die Wahrnehmung des Meinungsklimas}

Die in Abbildung 1 dargestellten Beziehungen zwischen den Einschätzungen zur derzeitigen Beliebtheit der Parteien, den Wahlerwartungen und den Quellen dieser Urteile haben wir auf Basis von Pfadmodellen getestet, die mithilfe des Programmpakets Mplus 7.0 durchgeführt wurden. Im Gegensatz zur Regressionsanalyse erlaubt ein Pfadmodell die parallele Schätzung des Einflusses unabhängiger Variablen auf mehrere abhängige. Außerdem ermöglicht es die Schätzung indirekter Effekte, die eine unabhängige Variable vermittelt über eine oder mehrere Mediatorvariablen ausübt - in unserem Fall beispielsweise den indirekten Einfluss der eigenen Meinung auf die Wahlerwartung, vermittelt über die Wahrnehmung der derzeitigen Beliebtheit einer Partei.

Die theoretisch angenommenen Wirkungszusammenhänge haben wir für insgesamt sieben zur Bundestagswahl angetretene Parteien geprüft, um auch eventuelle Unterschiede in den Urteilsstrukturen zwischen einzelnen Parteien zu identifizieren. Sämtliche dazu berechneten Modelle sind gerade identifiziert (saturierte Modelle). Das bedeutet, dass die in den Daten vorhandene Information (Varianzen, Kovarianzen) ausreicht, um die Modellparameter zu schätzen, nicht aber zur Berechnung von Modell-Fit-Indices. Die Beurteilung der Modellgüte erfolgt deshalb, wie in diesem Fall üblich, auf Basis der Pfadkoeffizienten sowie des Anteils erklärter Varianz $\left(\mathrm{R}^{2}\right)$ (Geiser 2010: $79 \mathrm{ff}$.).

\section{(1) Derzeitiges Meinungsklima (Beliebtheit der Parteien)}

Die individuellen Urteile über die momentane Beliebtheit der Parteien lassen sich durch die vier einbezogenen Faktoren unterschiedlich gut erklären. Dabei scheint die Erklärungskraft der Modelle zum einen mit der Größe der Parteien, zum anderen mit der Dauer zusammenzuhängen, die sie bereits Teil des Parteiensystems sind. Die Urteile über die Beliebtheit der großen Parteien Union $\left(\mathrm{R}^{2}=.09\right)$ und SPD $\left(\mathrm{R}^{2}\right.$ $=.13$ ) lässt sich nur vergleichsweise schlecht, die Beliebtheit von Bündnis 90/Die Grünen, FDP, Die Linke und Piraten sehr viel besser erklären ( $\mathrm{R}^{2}$ zwischen .19 und . 33). Noch höher ist die Varianzaufklärung bei der AfD, die erst kurz vor der Bun- 
destagswahl gegründet wurde $\left(\mathrm{R}^{2}=.49\right)$. Dies liegt vermutlich daran, dass die Wähler bei einer neuen Partei kaum andere als die einbezogenen Informationsquellen haben, um sich ein entsprechendes Urteil zu bilden. So können sie zum Beispiel nicht auf längerfristige Erfahrungen mit dem Image der Partei zurückgreifen.

Versucht man nun in einem ersten Schritt, über alle Parteien hinweg eine Hierarchie der Einflussfaktoren zu erstellen, erweisen sich die Wahrnehmung des Medientenors und der Meinungen im sozialen Umfeld als bedeutsamste Quellen für die Einschätzung des derzeitigen Meinungsklimas. Bei drei Parteien deutet sich dabei ein etwas stärkerer Einfluss des wahrgenommenen Medientenors an (Union, Linke, Piraten), in einem Fall des Umfelds (AfD) und bei drei Parteien sind die Effekte von Medientenor und Umfeld etwa gleich groß (SPD, Grüne, FDP). Das bedeutet: Je positiver die Berichterstattung und die Ansichten im sozialen Umfeld $\mathrm{zu}$ einer Partei wahrgenommen wurden, desto eher glaubten die Befragten auch an eine größere Beliebtheit in der gesamten Wählerschaft. Überraschenderweise sind signifikante Projektionseffekte nur bei der FDP und den Piraten zu beobachten. Das

Tabelle 2: Einflüsse auf die Wahrnehmung der derzeitigen Beliebtheit deutscher Parteien (Standardisierte Pfadkoeffizienten u. Standardfehler)

\begin{tabular}{|c|c|c|c|c|c|c|c|}
\hline & \multicolumn{7}{|c|}{$\begin{array}{l}\text { Wahrnehmung des derzeitigen Meinungsklimas } \\
\text { (derzeitige Beliebtheit der Parteien) }\end{array}$} \\
\hline & $\begin{array}{l}\text { Union } \\
(\mathrm{n}=568)\end{array}$ & $\begin{array}{c}\text { SPD } \\
(n=566)\end{array}$ & $\begin{array}{l}\text { Grüne } \\
(\mathrm{n}=51)\end{array}$ & $\begin{array}{c}\text { FDP } \\
(n=488)\end{array}$ & $\begin{array}{l}\text { Linke } \\
(\mathrm{n}=442)\end{array}$ & $\begin{array}{l}\text { Piraten } \\
(\mathrm{n}=309)\end{array}$ & $\begin{array}{c}\text { AfD } \\
(n=259)\end{array}$ \\
\hline \multicolumn{8}{|l|}{ Direkte Effekte } \\
\hline Eigene Meinung & $\begin{array}{l}.04 \\
(.05)\end{array}$ & $\begin{array}{l}.03 \\
(.05)\end{array}$ & $\begin{array}{l}.05 \\
(.05)\end{array}$ & $\begin{array}{l}.22 * * * \\
(.04)\end{array}$ & $\begin{array}{l}.02 \\
(.06)\end{array}$ & $\begin{array}{l}.20 * * * \\
(.06)\end{array}$ & $\begin{array}{l}.12 \\
(.07)\end{array}$ \\
\hline $\begin{array}{l}\text { Meinung im sozialen Um- } \\
\text { feld }\end{array}$ & $\begin{array}{l}.13^{* *} * \\
(.05)\end{array}$ & $\begin{array}{l}.21 * * * \\
(.05)\end{array}$ & $\begin{array}{l}.28 * * * \\
(.05)\end{array}$ & $\begin{array}{l}.27^{* * *} \\
(.04)\end{array}$ & $\begin{array}{l}.23 * * * \\
(.06)\end{array}$ & $\begin{array}{l}.20 * * * \\
(.06)\end{array}$ & $\begin{array}{l}.40 * * * \\
(.07)\end{array}$ \\
\hline $\begin{array}{l}\text { Tendenz der Berichter- } \\
\text { stattung }\end{array}$ & $\begin{array}{l}.22 * * * \\
(.04)\end{array}$ & $\begin{array}{l}.20 * * * \\
(.04)\end{array}$ & $\begin{array}{l}.25^{* * *} \\
(.04)\end{array}$ & $\begin{array}{l}.26^{* * * *} \\
(.04)\end{array}$ & $\begin{array}{l}.30 * * * \\
(.04)\end{array}$ & $\begin{array}{l}.31 * * * \\
(.05)\end{array}$ & $\begin{array}{l}.33 * * * \\
(.05)\end{array}$ \\
\hline $\begin{array}{l}\text { Erinnerte Umfrageergeb- } \\
\text { nisse }\end{array}$ & $\begin{array}{c}.04 \\
(.04)\end{array}$ & $\begin{array}{l}.09^{*} \\
(.04)\end{array}$ & $\begin{array}{l}.07 \\
(.04)\end{array}$ & $\begin{array}{l}-.03 \\
(.04)\end{array}$ & $\begin{array}{l}.15^{* * * *} \\
(.04)\end{array}$ & $\begin{array}{l}.05 \\
(.05)\end{array}$ & $\begin{array}{l}.08 \\
(.05)\end{array}$ \\
\hline \multicolumn{8}{|l|}{ Kontrollvariablen } \\
\hline Interesse BTW & $\begin{array}{l}.08^{*} \\
(.04)\end{array}$ & $\begin{array}{l}.02 \\
(.04)\end{array}$ & $\begin{array}{l}.01 \\
(.04)\end{array}$ & $\begin{array}{c}.00 \\
(.04)\end{array}$ & $\begin{array}{l}-.05 \\
(.04)\end{array}$ & $\begin{array}{l}-.11 * \\
(.05)\end{array}$ & $\begin{array}{l}-.04 \\
(.05)\end{array}$ \\
\hline Geschlecht (weibl.) & $\begin{array}{l}.01 \\
(.04)\end{array}$ & $\begin{array}{l}.07 \\
(.04)\end{array}$ & $\begin{array}{l}-.05 \\
(.04)\end{array}$ & $\begin{array}{c}.08 \\
(.04)\end{array}$ & $\begin{array}{l}.08 * \\
(.04)\end{array}$ & $\begin{array}{c}.07 \\
(.05)\end{array}$ & $\begin{array}{c}.05 \\
(.05)\end{array}$ \\
\hline Alter & $\begin{array}{l}-.08 \\
(.04)\end{array}$ & $\begin{array}{l}-.05 \\
(.04)\end{array}$ & $\begin{array}{l}-.05 \\
(.04)\end{array}$ & $\begin{array}{l}-.01 \\
(.04)\end{array}$ & $\begin{array}{c}.04 \\
(.04)\end{array}$ & $\begin{array}{l}-.08 \\
(.05)\end{array}$ & $\begin{array}{l}-.06 \\
(.05)\end{array}$ \\
\hline $\mathrm{R}^{2}$ & $.09 * * *$ & $.13 * * *$ & $.19 * * *$ & $.23 * * *$ & $.24 * * *$ & $.33 * * *$ & $.49^{* * *}$ \\
\hline
\end{tabular}

Anmerkungen: $* \mathrm{p}<.05 ; * * \mathrm{p}<.01 ; * * * \mathrm{p}<.001$ 
heißt, nur bei diesen beiden Parteien erweist sich die persönliche Meinung als relevant für die Einschätzung der momentanen Beliebtheit. Ebenso bemerkenswert ist, dass sich die erinnerten Umfrageergebnisse als schwächster aller einbezogenen Prädiktoren erweisen, obwohl sie eigentlich die objektivste und unmittelbarste Information für die derzeitige Bevölkerungsmeinung darstellen. Relevant sind sie jedoch nur für die Einschätzung der SPD und der Linken (Tabelle 2).

\section{(2) Zukünftiges Meinungsklima (Wahlerwartungen)}

Unsere Hypothesen zu den Einflüssen auf die Wahrnehmung des künftigen Meinungsklimas (Wahlerwartungen) prüfen wir anhand zweier abhängiger Variablen. Zunächst geht es darum, welche Zweitstimmenanteile die Befragten für die Parteien erwarteten. Dann widmen wir uns der Frage, ob die Kleinparteien nach Ansicht der Befragten in den Bundestag einziehen würden. Dies ist natürlich besonders für die Parteien interessant ist, bei denen die Möglichkeit bestand, dass sie entweder knapp in den Bundestag einziehen oder knapp an der Fünf-Prozent-Hürde scheitern würden.

\section{Erwartete prozentuale Stimmenanteile}

Im Rahmen der Befragung baten wir die Teilnehmer zu schätzen, welchen Zweitstimmenanteil die Parteien bei der bevorstehenden Bundestagswahl erreichen würden. Analog zu den vorhergehenden Analysen zur derzeitigen Beliebtheit interessieren wir uns jetzt dafür, wie gut die von uns einbezogenen Faktoren diese Form der Wahlerwartung erklären können. Im Gegensatz zu den vorherigen Analysen kommt außerdem die Wahrnehmung des derzeitigen Meinungsklimas als weiterer möglicher Erklärungsfaktor hinzu. Die Befunde zeigen, dass die Modelle die Wahlerwartungen sogar noch besser erklären können als die Wahrnehmung der derzeitigen Beliebtheit $\left(\mathrm{R}^{2}\right.$ zwischen .20 und .47$) .^{5}$

Betrachtet man zunächst die direkten Effekte, ergibt sich für die Wahlerwartungen ein völlig anderes Einflussmuster als bei der Wahrnehmung der derzeitigen Beliebtheit der Parteien. Der wahrgenommene Medientenor übt nun nur noch in zwei Fällen (Union und SPD), die Wahrnehmung des sozialen Umfelds nur noch bei Union, SPD und Piraten einen signifikanten, jedoch nur sehr schwachen Einfluss auf die Prognose des Zweitstimmenergebnisses aus. Projektionseffekte zeigen sich in drei Fällen (Union, Grüne, FDP). Nur bei diesen beeinflusste also die eigene Meinung die Erwartungen an ihr Abschneiden am Wahltag.

5 Vergleiche hierfür und im Folgenden Tabelle 3. 
Als wichtigste Prädiktoren für die Wahlerwartungen der Bürger erweisen sich allerdings die erinnerten Umfragewerte, die bei allen Parteien einen mittleren bis starken Einfluss ausüben. Je höher die jeweiligen Umfragewerte, desto höhere Werte prognostizierten die Befragten für die Wahlergebnisse der jeweiligen Parteien. Schließlich ergeben sich auch noch signifikante Einflüsse der Wahrnehmung der derzeitigen Beliebtheit der Parteien, die wir als zusätzlichen Faktor in die Analysen aufgenommen hatten. Diese sind zwar nur bei FDP und AfD zu beobachten. Allerdings sind sie gerade bei der AfD vergleichsweise stark. Gleichzeitig sind bei FDP und AfD die Effekte der erinnerten Umfragewerte am geringsten. Dies bedeutet, dass sich in beiden Fällen die Erwartungen an ihr Abschneiden am stärksten von den erinnerten Umfragen lösten und andere Faktoren Einfluss gewannen. Das ist auch deshalb interessant, weil gerade mit Blick auf diese beiden Parteien ausführlich diskutiert wurde, ob ein Einzug bzw. ein Ausscheiden aus dem deutschen Bundestag möglich ist. Wir werden dieser Art der Wahlerwartung im nächsten Abschnitt noch weiter nachgehen.

Aus den Pfadanalysen ergibt sich weiterhin, dass die Erwartungen an das Abschneiden von FDP und AfD auch indirekten Einflüssen unterlagen, die über die wahrgenommene Beliebtheit der Parteien vermittelt wurden. Für die FDP sind indirekte Effekte der eigenen Meinung, des sozialen Umfelds und der Tendenz der Medienberichterstattung feststellbar. Bemerkenswert ist dabei, dass die Wahrnehmung der Meinungen im sozialen Umfeld und des Medientenors ausschließlich über die wahrgenommene Beliebtheit der FDP auf die Wahlerwartungen wirken. Für die AfD ergeben sich ebenfalls indirekte Einflüsse der sozialen Umwelt und der Berichterstattung, wobei auch hier beide Größen nur vermittelt über die wahrgenommene Beliebtheit der Parteien wirken.

\section{Erwartungen zum Einzug der Kleinparteien ins Parlament}

Meinungsklimawahrnehmungen besitzen besondere Relevanz, wenn sie Grundlage von Wahlentscheidungen werden und so mittelbar weiterreichende politische Folgen haben, zum Beispiel den Einzug bzw. das Ausscheiden bestimmter Parteien aus dem Parlament. In den Wochen vor der Bundestagswahl 2013 wurden für FDP und AfD stets Umfragewerte nahe der Fünf-Prozent-Hürde publiziert, die Piraten lagen dagegen meist deutlich darunter. Entsprechend konzentrierte sich die öffentliche Diskussion hauptsächlich auf ein mögliches Ausscheiden der FDP bzw. einen möglichen Einzug der AfD, wohingegen die Chancen der Piraten kaum thematisiert wurden. 
Wir haben daher speziell für die kleineren Parteien im Bund untersucht, welche Faktoren zum Eindruck beitrugen, ob sie den Einzug in den Bundestag schaffen würden oder nicht. Tabelle 3 zeigt analog zu den bisherigen Analysen die Gewichte, mit denen die einzelnen Wahrnehmungsquellen in die diesbezüglichen Erwartungen unserer Befragten eingehen. Zunächst fällt auf, dass die von uns theoretisch angenommenen Informationsquellen kaum in der Lage sind, die entsprechenden Wahlerwartungen der Bürger zu klären - mit Ausnahme der Piraten und der AfD $\left(\mathrm{R}^{2}\right.$ Piraten $\left.=.29 ; \mathrm{R}_{\mathrm{AfD}}^{2}=.46\right)$, was vor allem darauf zurückzuführen ist, dass in beiden Fällen die erinnerten Umfragewerte die Erwartungen der Befragten maßgeblich prägen. Außerdem zeigen sich in beiden Fällen auch andere Wahrnehmungsquellen als bedeutsam. Bei den Piraten spielt die wahrgenommene Meinung im persönlichen Umfeld eine Rolle, hinsichtlich der AfD die eigene Meinung und die Einschätzung ihrer derzeitigen Beliebtheit - ein Faktor, der bei den anderen Parteien, mit Ausnahme der Grünen, völlig bedeutungslos ist. Der Einfluss der derzeitigen Beliebtheit führt wiederum dazu, dass auch die im persönlichen Umfeld wahrgenommenen Meinungen zur AfD und der Medientenor indirekte Effekte auf die Wahlerwartungen ausüben. Zusätzlich lässt sich außerdem ein leichter Projektionseffekt feststellen: Befragte, die eine gute Meinung von der AfD hatten, sahen sie auch tendenziell eher im Parlament, solche mit einer schlechten Meinung hingegen eher nicht. Im Gegensatz dazu scheinen die Erwartungen an einen Einzug der Grünen und der FDP völlig von den unterstellten Einflussgrößen abgekoppelt. Im Fall der FDP erweist sich lediglich die eigene Meinung als signifikanter Prädiktor, bei den Grünen die Einschätzung ihrer allgemeinen derzeitigen Beliebtheit, wobei der Einfluss in beiden Fällen eher schwach ausfällt. Die Erwartungen an einen Einzug bzw. Verbleib der Linken war hingegen ausschließlich von den erinnerten Umfragewerten abhängig.

Sicherlich lassen sich die fehlenden Effekte bei einigen Parteien, vor allem den Grünen und der Linken, durch eine fehlende Varianz in der abhängigen Variable erklären. Mit anderen Worten ging die Mehrzahl der Befragten davon aus, dass beide Parteien die Fünf-Prozent-Hürde sicher überspringen würden. So lag unter Umständen ein Einfluss veröffentlichter Umfragen vor, der allerdings statistisch nicht nachweisbar ist. Wesentlich überraschender ist allerdings die Entkopplung der FDP von sämtlichen Wahrnehmungsquellen. Obwohl die Sicherheit eines Verbleibs im Bundestag keineswegs gegeben war und die Befragten hier auch zu höchst unterschiedlichen Urteilen kamen, scheint diese Einschätzung andere Ursachen als die von uns angenommenen zu haben. Man kann aber vermuten, dass hier längerfristig über viele Jahre und Wahlen entstandene Erwartungen den Einfluss kurzfristiger Wahrnehmungen überlagern, nach dem Motto: Die FDP hat es noch immer geschafft, dann schafft sie es auch diesmal (vgl. Tabelle 3). 
Die Wahrnehmung des Meinungsklimas im Bundestagswahlkampf 2013

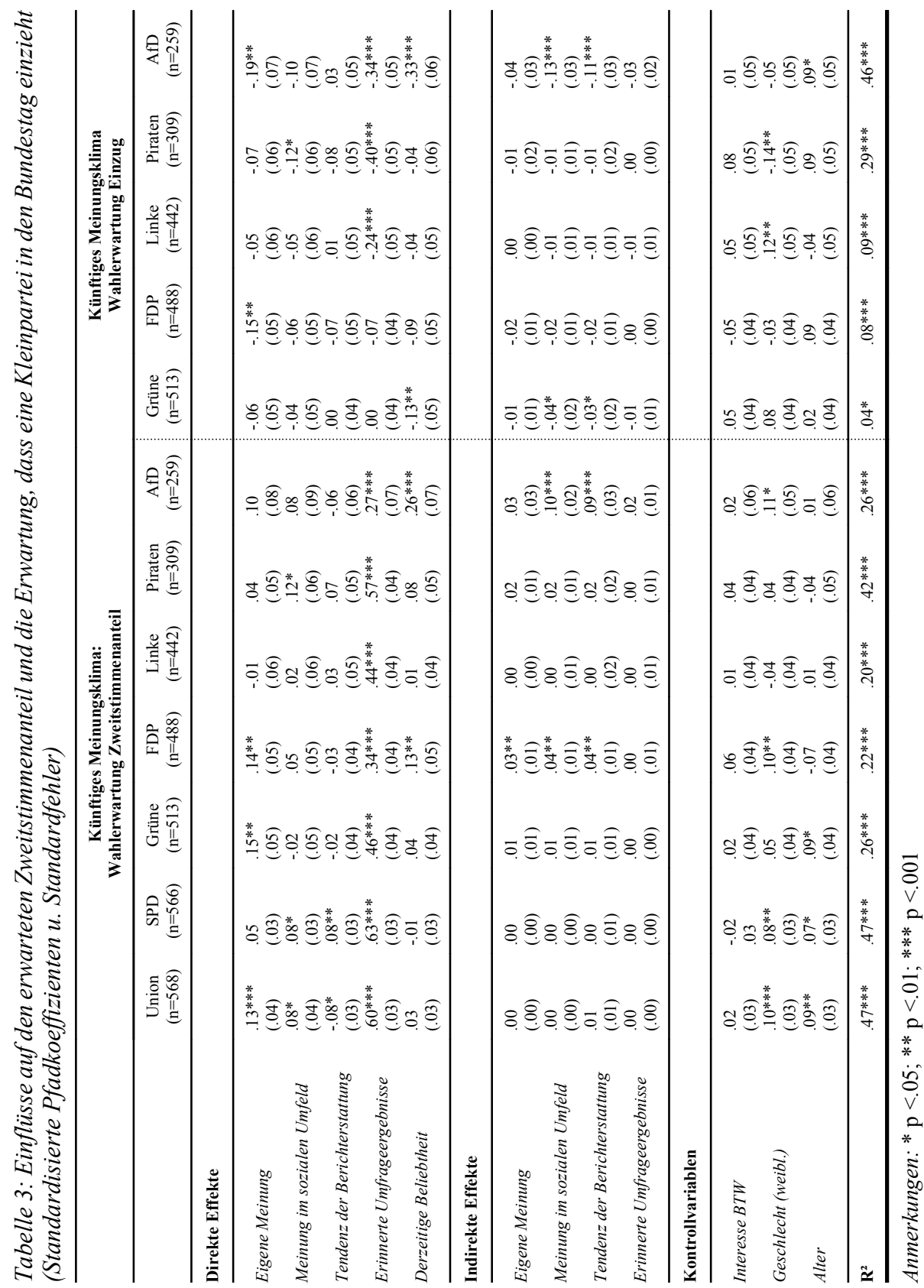


Die ausgewiesenen indirekten Effekte ergeben sich aus der Multiplikation der Koeffizienten entlang des entsprechenden Modellpfads. Da das Produkt von Koeffizienten nicht mehr normalverteilt ist, beruht die Signifikanzprüfung auf einem korrigierten Test, der dies berücksichtigt (BC bootstrap) (Cheung/Lau 2008; Holbert/Stephenson 2003; MacKinnon/Lockwood/Williams 2004).

\section{Zusammenfassung und Diskussion}

Der vorliegende Beitrag untersuchte die Frage, wie Urteile über die gegenwärtige Beliebtheit von Parteien (derzeitiges Meinungsklima) und Erwartungen an den Ausgang einer Wahl (künftiges Meinungsklima) entstehen. In der Wahlforschung gelten solche Urteile als relevant, weil sie zumindest potenziell die Motivation zur Wahlteilnahme oder sogar die Wahlentscheidung selbst beeinflussen können. Als mögliche Einflüsse auf Meinungsklimawahrnehmungen werden in der Literatur in erster Linie eigene Meinungen (Projektionseffekte), das soziale Umfeld, aktuelle Umfragen und der Tenor der Medienberichterstattung genannt. Allerdings entstammt die bisherige Literatur zum Thema sehr unterschiedlichen Forschungsansätzen aus Sozialpsychologie, Kommunikations- und Politikwissenschaft. Dies hat unter anderem zur Folge, dass die verschiedenen möglichen Ursachen von Meinungsklima-Einschätzungen bzw. Wahlerwartungen meist nicht in ihrer Gesamtheit betrachtet werden. Wenn etwa mediale Einflüsse auf das wahrgenommene Meinungsklima untersucht werden, beschränkt sich dies meist auf medial vermittelte Umfragen, während der Tenor der Berichterstattung unberücksichtigt bleibt. Im Kontext von Studien zu Wahlerwartungen werden neben Umfragen auch eigene Einstellungen einbezogen, während das soziale Umfeld in der Regel ausgeblendet wird. Auch bleiben in bisherigen Untersuchungen die Beziehungen zwischen der Wahrnehmung des derzeitigen und des künftigen Meinungsklimas weitgehend im Dunkeln.

Den genannten Defiziten begegnete die vorliegende Studie, indem sie erstmals die wichtigsten bislang in der Literatur berücksichtigten Ursachen für die Wahrnehmung des derzeitigen und des künftigen Meinungsklimas einbezog und ihren Einfluss im Vergleich zueinander für sieben bei der Bundestagswahl 2013 angetretene Parteien untersuchte. Die Basis der Analyse bildete eine für Berlin repräsentative Telefonbefragung zwei Wochen vor der Bundestagswahl 2013. Um den Einfluss der einbezogenen Merkmale herauszuarbeiten, wurden die Hypothesen mittels Pfadanalysen geprüft. Die Befunde lassen sich folgendermaßen zusammenfassen: 1. Die Wahrnehmung des derzeitigen Meinungsklimas (Beliebtheit der Parteien) wird maßgeblich vom wahrgenommenen Medientenor und den Meinungen im sozialen Umfeld bestimmt. Die Hypothesen 4 a und 5 a bestätigen sich somit in allen 
Fällen. Anders als oftmals angenommen, spielten Umfragewerte und die eigene Meinung nur bei einzelnen Parteien eine Rolle. Die Hypothesen 2 a und 3 a können nur in zwei von sieben Fällen bestätigt werden. Die Wähler leiteten ihre Urteile also vornehmlich aus meinungshaltigen Informationen ihrer unmittelbaren und massenmedialen sozialen Umwelt ab. Vermutlich erlauben Umfragewerte als solche entsprechende Urteile über die soziale Umwelt nur begrenzt, da sie in der Regel der Einordnung bedürfen. Den meisten Menschen dürfte es schlicht schwer fallen, aus nackten Zahlen die Beliebtheit einer Partei abzuleiten. Dies scheint für viele nur bei sehr hohen oder sehr niedrigen Umfragewerten möglich zu sein. Oftmals erfordern Umfragen dagegen vermutlich eine Interpretation durch Dritte, um sie als Anker für entsprechende Urteile nutzen zu können. Das weitgehende Ausbleiben von Projektionseffekten deutet darauf hin, dass diese vermutlich in all denjenigen Studien überschätzt werden, die soziale Indikatoren wie das persönliche Umfeld in ihren Analysen nicht kontrollieren. Denn es scheint so zu sein, dass Urteile über das soziale Phänomen „derzeitiges Meinungsklima“ tatsächlich vornehmlich durch soziale Wahrnehmungen geprägt sind. Die in der Literatur beobachteten Projektionseffekte dürften daher zumindest teilweise durch soziale Selektion entstehen, also dadurch, dass Meinungen im sozialen Umfeld oft relativ homogen sind und der eigenen Meinung entsprechen, was sie für Individuen kognitiv sehr salient macht (Marks/Miller 1987) (Abbildung 2).

2. Ein völlig anderes Einflussmuster als bei den Urteilen über das derzeitige zeigt sich bei den Erwartungen an das künftige Meinungsklima (Wahlerwartungen). Der nahezu völlig dominierende Faktor sind hier die erinnerten Umfrageergebnisse. Hypothese 3 b bestätigt sich für die Schätzung des Wahlergebnisses für alle Parteien. Die anderen möglichen Quellen sind dagegen nur bei einzelnen Parteien bedeutsam und die Effekte fallen in den meisten Fällen auch sehr viel schwächer aus als die der erinnerten Umfragen. Die Analysen erbringen für die Hypothesen 2b, 4b und $5 \mathrm{~b}$ nur in zwei bzw. drei Fällen eine Bestätigung. Dieses Einflussmuster zeigt sich mit Einschränkungen auch für die Erwartung des Einzugs der Kleinparteien. Diese Ergebnisse sprechen gegen eine generelle parteiübergreifende Bedeutung von Projektionseffekten (,,wishful thinking"), die wirklich aus der eigenen Meinung der Befragten resultieren, gegen eine dominierende Bedeutung des sozialen Umfelds und auch gegen die These einer generellen „Persuasive Press Inference“ (PPI) im Zusammenhang mit der Erklärung von Wahlerwartungen. Bemerkenswert ist darüber hinaus, dass die wahrgenommene Beliebtheit der Parteien zumindest bei einzelnen Parteien einen Effekt auf die Wahlerwartungen ausübt und Hypothese 1 deshalb für die Schätzung des Wahlergebnisses für zwei Parteien nicht zurückgewiesen werden muss. Dies bedeutet gleichzeitig, dass in diesen Fällen auch indirekte Ef- 
fekte der eingangs einbezogenen Quellen der Meinungsklima-Wahrnehmungen festzustellen sind (Abbildung 2).

Abbildung 2: Anzahl der bestätigten Hypothesen im Parteienvergleich (ohne Erwartungen an den Einzug in den Bundestag)

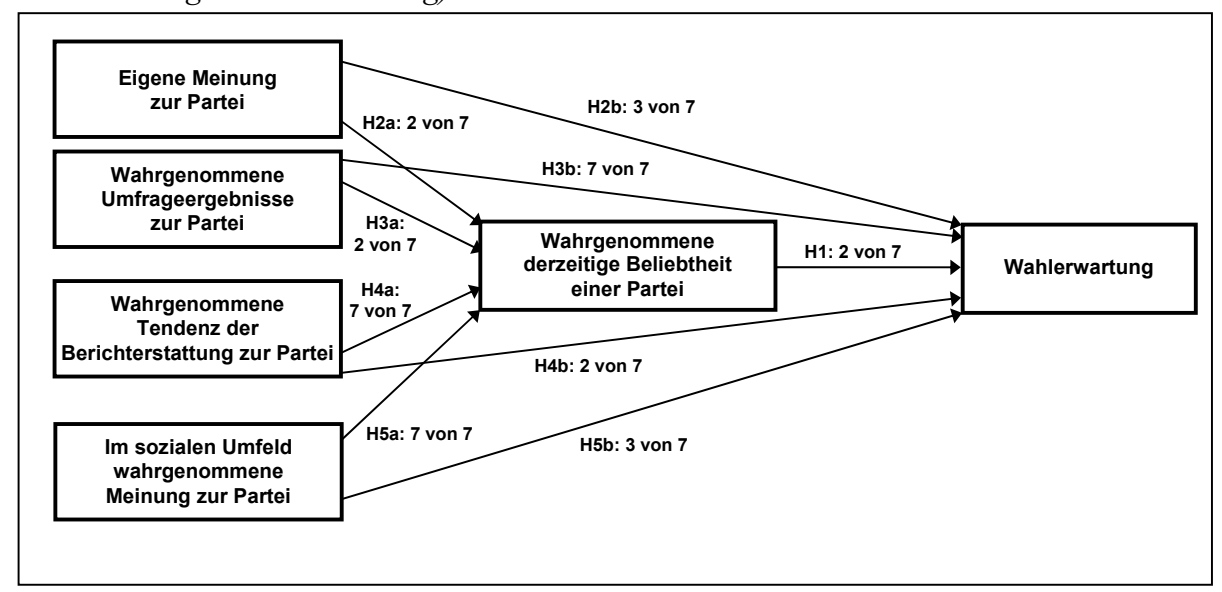

Anmerkung: Die Pfade repräsentieren die theoretisch unterstellten Hypothesen. Die Beschriftung der Pfade gibt an, wie oft sich die jeweilige Hypothese bei insgesamt sieben Parteien bestätigt hat. Nicht ausgewiesen sind die indirekten Effekte sowie die Befunde zu den Erwartungen an den Einzug der Kleinparteien in den Bundestag.

3. Neben den großen Gemeinsamkeiten in den Einflussstrukturen über die Parteien hinweg werden jedoch auch Unterschiede zwischen den Parteien erkennbar. Sie beziehen sich sowohl darauf, wie gut die einbezogenen Faktoren die Meinungsklima-Wahrnehmungen insgesamt erklären können, als auch auf vereinzelte Effekte von Einflussgrößen, die man insgesamt als eher weniger bedeutend einstufen muss. Diese singulären Effekte legen nahe, dass Einschätzungen und Erwartungen des Meinungsklimas im Einzelfall sehr wohl auch von den Merkmalen der Parteien bzw. dem situativen Kontext einer Wahl abhängen. Im Wahlkampf 2013 scheinen dabei die Größe der Parteien, ihre Etablierung im Parteiensystem sowie die Frage, ob für sie ein mögliches Scheitern oder Überspringen der Fünf-Prozent-Hürde diskutiert bzw. erwartet wurde, von Bedeutung gewesen zu sein.

So lässt sich die Wahrnehmung der Beliebtheit und der Wahlchancen der AfD durch die berücksichtigten Faktoren besonders gut erklären. Dies liegt vermutlich daran, dass die Wähler für die AfD als gerade gegründete Partei noch keine Erfahrungswerte in Form von Wahlergebnissen und langfristigen Umfrageentwicklungen hatten, auf die sie bei ihren Urteilen zurückgreifen konnten. Im Gegensatz dazu 
koppelten sich die Erwartungen an den Einzug der FDP völlig von den erinnerten Umfragewerten $a b$. Vermutlich griffen die Befragten hier anders als bei der AfD auf ihre langjährigen Erfahrungen mit der Partei zurück, die bislang noch immer den Einzug in den Bundestag geschafft hatte. Diese Erfahrungswerte führten im Ergebnis möglicherweise zu einer kollektiven Täuschung über die Chancen der FDP, den Sprung in den Bundestag zu schaffen.

Der Befund, dass abgesehen von der FDP gerade bei Parteien nahe der FünfProzent-Hürde die erinnerten Umfrageergebnisse maßgeblich die Erwartung prägen, ob diese ins Parlament einziehen, macht zudem auch Umfrageeffekte auf das Wahlverhalten in entsprechenden Situationen wahrscheinlicher. Die Wähler scheinen unter diesen Umständen Umfrageergebnisse stärker in ihre Urteile einzubeziehen. Ob sie dann tatsächlich verhaltensrelevant werden, wird allerdings davon abhängen, ob sie auf Basis der Umfragen den Einzug der entsprechenden Partei erwarten oder nicht. Die Tatsache, dass bei einigen Parteien durchaus ein beachtlicher Einfluss der eigenen Meinung zu beobachten war, deutet ebenfalls auf komplexere und partei- bzw. situationsabhängige Urteilsstrukturen hin. Möglicherweise verlassen sich Anhänger einer Partei, über die negativ berichtet oder die durch das soziale Umfeld schlecht bewertet wird, eher auf ihre persönliche Einstellung oder neigen zu einem „optimistic bias“ oder eben „wishful thinking“. Dies gilt es in weiteren Analysen der vorliegenden Daten näher zu untersuchen.

Wie jede empirische Untersuchung unterliegt auch die vorliegende gewissen Einschränkungen. So muss weiter untersucht werden, inwieweit die ermittelten Einflussstrukturen wahlspezifisch sind oder sich auf andere Wahlkämpfe übertragen lassen. Auch themenspezifische Urteilsstrukturen sind durchaus denkbar (Shamir/ Shamir 2000). Die Begrenzung der Befragung auf Berlin schränkt die Aussagekraft der Studie unseres Erachtens dagegen kaum ein, da es hier nicht um bevölkerungsrepräsentative Aussagen über die Wahrnehmungen der deutschen Wähler ging, sondern um die theoriegeleitete Untersuchung von Zusammenhängen zwischen meinungsklima-relevanten Wahrnehmungen und Urteilen, die unabhängig von der gewählten Stichprobe bestehen sollen. Auch die besondere politische Struktur der Berliner Wählerschaft stellt insofern kein Problem dar, sondern erlaubt vielmehr durch die bestehende Varianz in den Urteilen auch die Untersuchung bundesweit eher kleinerer Parteien. Betonen muss man allerdings, dass hier allein die Wahrnehmung von Meinungen im sozialen Umfeld untersucht wurde. Obwohl man argumentieren kann, dass es genau diese subjektiven Wahrnehmungen sein dürften, die mögliche Verhaltenswirkungen nach sich ziehen, ist damit beispielsweise die Frage nach den Wirkungen der Medienberichterstattung oder der Umfragen als solcher noch nicht beantwortet. Dazu muss man vielmehr der Frage nachgehen, mit 
welchen Medieninhalten und Umfragedaten die Wähler tatsächlich konfrontiert wurden.

Ein weiterer Punkt betrifft die Breite der hier verwendeten Skalen: So könnte sich die Verwendung einer fünfstufigen Skala zur Bestimmung der wahrgenommenen Beliebtheit der Parteien auf die Stärke der Zusammenhänge mit den beiden prozentualen Skalen ausgewirkt haben, da die Befragten je nach Skala unterschiedlich differenziert antworten konnten. Die vergleichsweise schmale Skala dürfte im Vergleich zu einer breiteren (z. B. elfstufigen Skala) eher dazu geführt haben, dass die Zusammenhänge unterschätzt werden.

Kritisch zu reflektieren ist ferner die in der vorliegenden Studie vorgenommene Operationalisierung des derzeitig und zukünftig wahrgenommenen Meinungsklimas gegenüber den Parteien als Einschätzung ihrer momentanen Beliebtheit bzw. ihres potenziellen Wahlerfolgs. So erklärt sich beispielsweise ein Teil des starken Umfrageeinflusses auf die Wahlerwartungen sicherlich durch die formale Ähnlichkeit der beiden prozentualen Antwortskalen. Allerdings lässt sich der Umfrageeinfluss auch bei der Bewertung der Wahrscheinlichkeit zum Einzug der kleinen Parteien ins Parlament beobachten, die lediglich mit einer fünfstufigen Skala abgefragt wurde. Es handelt sich daher bei den Umfrageeffekten auf das künftige Meinungsklima nicht um ein bloßes Messartefakt. Darüber hinaus dürften aber auch inhaltliche Unterschiede zwischen beiden Konstrukten zu den gefundenen bzw. ausbleibenden Zusammenhängen beigetragen haben. So ergeben sich die unterschiedlichen Einflussstärken der einzelnen Informationsquellen vermutlich auch deshalb, weil in die Ausbildung einer Wahlerwartung auch langfristigere Faktoren und Überlegungen eingehen. Dazu gehören vor allem die vergangenen Wahlerfolge einer Partei bzw. die Wahrnehmung langfristig bestehender Parteibindungen. Dies würde auch erklären, warum unsere Modelle insbesondere bei den neu hinzugekommenen Parteien wie AfD und Piraten eine deutlich größere Erklärungskraft aufweisen als dies bei den etablierten Parteien der Fall ist.

Trotz der genannten Einschränkungen hat die vorliegende Studie gezeigt, dass die Entstehung von Meinungsklima-Einschätzungen und Wahlerwartungen offenbar komplexer und in Teilen auch anders zu erklären ist, als dies die bisherige Forschung nahelegt. Die entsprechenden Prozesse und vor allem auch deren Folgen für das Wahlverhalten gilt es nun weiter zu untersuchen. 
Die Wahrnehmung des Meinungsklimas im Bundestagswahlkampf 2013

\section{Literatur}

Babad, Elisha/Hills, Michael/O'Driscoll, Michael, 1992: Factors influencing wishful thinking and predictions of election outcomes, in: Basic and Applied Social Psychology 13 (4), 461-476.

Bargsted, Matias A./Kedar, Orit, 2009: Coalition-targeted duvergerian voting: How expectations affect voter choice under proportional representation, in: American Journal of Political Science 53 (2), 307-323.

Blais, Andre/Bodet, Marc Andre, 2006: How do voters form expectations about the parties' chances of winning the election?, in: Social Science Quarterly 87 (3), 477-493.

Blais, André/Gidengil, Elisabeth/Fournier, Patrick/Nevitte, Neil/Hicks, Bruce M., 2008: Measuring expectations: Comparing alternative approaches, in: Electoral Studies 27 (2), 337-343.

Brettschneider, Frank, 2008: The news media's use of public opinion polls, in: Wolfgang Donsbach/Michael W. Traugott (Hrsg.), The SAGE handbook of public opinion research, Los Angeles/London, 479-486.

Brosius, Hans-Bernd/Bathelt, Anke, 1994: The utility of exemplars in persuasive communications, in: Communication Research 21 (1), 48-78.

Cheung, Gordon W./Lau, Rebecca S., 2008: Testing mediation and suppression effects of latent variables: Bootstrapping with structural equation models, in: Organizational Research Methods 11 (2), 296-325.

Christen, Cindy T./Gunther, Albert C., 2003: The influence of mass media and other culprits on the projection of personal opinion, in: Communication Research 30 (4), 414-431.

Christen, Cindy T./Kannaovakun, Prathana/Gunther, Albert C., 2002: Hostile media perceptions: Partisan assessments of press and public during the 1997 United Parcel Service strike, in: Political Communication 19 (4), 423-436.

Daschmann, Gregor, 2001: Der Einfluss von Fallbeispielen auf Leserurteile. Experimentelle Untersuchungen zur Medienwirkung, Konstanz.

Davison, W. Phillips, 1958: The public opinion process, in: Public Opinion Quarterly 22 (2), 91-106.

Davison, W. Phillips, 1983: The third-person effect in communication, in: Public Opinion Quarterly 47 (1), 1-15.

Delavande, Adeline/Manski, Charles F., 2012: Candidate preferences and expectations of election outcomes, in: Proceedings of the National Academy of Sciences 109 (10), 3711-3715. 
Donsbach, Wolfgang/Weisbach, Kerstin, 2005: Kampf um das Meinungsklima. Quellen der Aussagen über den möglichen Wahlausgang, in: Elisabeth NoelleNeumann/Wolfgang Donsbach/Hans Mathias Kepplinger (Hrsg.), Wählerstimmungen in der Mediendemokratie. Analysen auf der Basis des Bundestagswahlkampfs 2002, Freiburg, 104-127.

Faas, Thorsten/Schmitt-Beck, Rüdiger, 2007: Wahrnehmung und Wirkungen politischer Meinungsumfragen. Eine Exploration zur Bundestagwahl 2005, in: Frank Brettschneider/Oskar Niedermayer/Bernhard Wessels (Hrsg.), Die Bundestagswahl 2005. Analysen des Wahlkampfes und der Wahlergebnisse, Wiesbaden, 233-267.

Fields, James M./Schuman, Howard, 1976: Public beliefs about the beliefs of the public, in: Public Opinion Quarterly 40 (4), 427-448.

Gabler, Siegfried/Häder, Sabine, 1997: Überlegungen zu einem Stichprobendesign für Deutschland, in: ZUMA-Nachrichten 41, 7-18.

Geiser, Christian, 2010: Datenanalyse mit Mplus: Eine anwendungsorientierte Einführung. Wiesbaden (1. Aufl.).

Gimpel, James G./Harvey, Diane Hollern, 1997: Forecasts and preferences in the 1992 general election, in: Political Behavior 19 (2), 157-175.

Granberg, Donald/Brent, Edward, 1983: When prophecy bends: The preferenceexpectation link in U.S. presidential elections, 1952-1980, in: Journal of Personality and Social Psychology 45 (3), 477-491.

Gunther, Albert C., 1998: The persuasive press inference: Effects of mass media on perceived public opinion, in: Communication Research 25 (5), 486-504.

Gunther, Albert C./Christen, Cindy T., 1999: Effects of news slant and base rate information on perceived public opinion, in: Journalism \& Mass Communication Quarterly 76 (2), 277-292.

Gunther, Albert C./Christen, Cindy T., 2002: Projection or persuasive press? Contrary effects of personal opinion and perceived news coverage on estimates of public opinion, in: Journal of Communication 52 (1), 177-195.

Gunther, Albert C./Christen, Cindy T./Liebhart, Janice L./Chia, Stella Chih-Yun, 2001: Congenial public, contrary press, and biased estimates of the climate of opinion, in: Public Opinion Quarterly 65 (3), 295-320.

Gunther, Albert C./Storey, J. Douglas, 2003: The influence of presumed influence, in: Journal of Communication 53 (2), 199-215.

Hoffmann, Hanna/Klein, Markus, 2013: Wirkungen von veröffentlichten Wahlumfragen auf die Koalitionserwartung, die Wahlbeteiligung und die Wahlentscheidung bei der Bundestagswahl 2009: Eine Mehrebenenanalyse auf der Grundlage der Rolling Cross-Section-Befragung der German Longitudinal Election Study 
(GLES), in: Thorsten Faas/Kai Arzheimer/Sigrid Roßteutscher/Bernhard Weßels (Hrsg.), Koalitionen, Kandidaten, Kommunikation, Wiesbaden, 221-246.

Holbert, R. Lance/Stephenson, Michael T., 2003: The importance of indirect effects in media effects research: Testing for mediation in structural equation modeling, in: Journal of Broadcasting \& Electronic Media 47 (4), 556-572.

Huber, Brigitte, 2011: Experten oder Ersatzjournalisten? Zur Rolle der Meinungsforscher in der Wahlkampfberichterstattung, in: Josef Ferdinand Haschke/Andre Michael Moser (Hrsg.), Politik - Deutsch, Deutsch - Politik. Aktuelle Trends und Forschungsergebnisse: Beiträge zur 6. Fachtagung des DFPK, Berlin, 113-132.

Huber, Sascha/Gschwend, Thomas/Meffert, Michael F./Pappi, Franz Urban, 2009: Erwartungsbildung über den Wahlausgang und ihr Einfluss auf die Wahlentscheidung, in: Oscar W. Gabriel/Bernhard Wessels/Jürgen W. Falter (Hrsg.), Wahlen und Wähler. Analysen aus Anlass der Bundestagswahl 2005, Wiesbaden, 561-584.

Irwin, Galen A./van Holsteyn, Joop J. M., 2002: According to the polls: The influence of opinion polls on expectations, in: Public Opinion Quarterly 66 (1), 92-104.

Kahneman, Daniel/Tversky, Amos, 1973: On the psychology of prediction, in: Psychological Review 80 (4), 237-251.

Katz, Elihu, 1982: Publicity and pluralistic ignorance: Notes on 'The Spiral of Silence', in: Horst Baier/Hans Mathias Kepplinger/Kurt Reumann (Hrsg.), Öffentliche Meinung und sozialer Wandel / Public opinion and social change, Wiesbaden, 28-38.

Krizan, Zlatan/Miller, Jeffrey C./Johar, Omesh, 2010: Wishful thinking in the 2008 U.S. presidential election, in: Psychological Science 21 (1), 140-146.

Krizan, Zlatan/Sweeny, Kate, 2013: Causes and consequences of expectation trajectories: "High" on optimism in a public ballot Initiative, in: Psychological Science 24 (5), 706-714.

Krizan, Zlatan/Windschitl, Paul D., 2007: The influence of outcome desirability on optimism, in: Psychological Bulletin 133 (1), 95-121.

Lewis-Beck, Michael S./Skalaban, Andrew, 1989: Citizen forecasting: Can voters see into the future?, in: British Journal of Political Science 19 (1), 419-427.

Lewis-Beck, Michael/Tien, Charles, 1999: Voters as forecasters: A micromodel of election prediction, in: International Journal of Forecasting 15 (2), 175-184. 
MacKinnon, David P./Lockwood, Chondra M./Williams, Jason, 2004: Confidence limits for the indirect effect: Distribution of the product and resampling methods, in: Multivariate Behavioral Research 39 (1), 99-128.

Marks, Gary/Miller, Norman, 1987: Ten years of research on the false-consensus effect: An empirical and theoretical review, in: Psychological Bulletin 102 (1), 72-90.

Marsh, Catherine, 1985: Back on the bandwagon: The effect of opinion polls on public opinion, in: British Journal of Political Science 15 (1), 51-74.

Mayer, Russell K., 2001: What to expect from electoral expectations, in: The Harvard International Journal of Press/Politics 6 (3), 71-89.

Meffert, Michael F./Huber, Sascha/Gschwend, Thomas/Pappi, Franz Urban, 2011: More than wishful thinking: Causes and consequences of voters' expectations about election outcomes, in: Electoral Studies 30 (4), 804-815.

Miller, Michael K./Wang, Guanchun/Kulkarni, Sanjeev R./Poor, H. Vincent/Osherson, Daniel N., 2012: Citizen forecasts of the 2008 U.S. presidential election, in: Politics \& Policy 40 (6), 1019-1052.

Morwitz, Vicki G./Pluzinski, Carol, 1996: Do polls reflect opinions or do opinions reflect polls? The impact of political polling on voters' expectations, preferences, and behavior, in: Journal of Consumer Research 23 (1), 53-67.

Mullen, Brian/Atkins, Jennifer L./Champion, Debbie S./Edwards, Cecelia/Hardy, Dana/Story, John E./Vanderklok, Mary, 1985: The false consensus effect: A meta-analysis of 115 hypothesis tests, in: Journal of Experimental Social Psychology 21 (3), 262-283.

Mutz, Diana C., 1995: Effects of horse-race coverage on campaign coffers: Strategic contributing in presidential primaries, in: The Journal of Politics 57 (4), 1015-1042.

Mutz, Diana C., 1997: Mechanisms of momentum: Does thinking make it so?, in: Journal of Politics 59 (1), 104-125.

Mutz, Diana C., 1998: Impersonal influence: How perceptions of mass collectives affect political attitudes, Cambridge.

Mutz, Diana C., 2006: Hearing the other side: Deliberative versus participatory democracy, New York.

Mutz, Diana C./Soss, Joe, 1997: Reading public opinion: The influence of news coverage on perceptions of public sentiment, in: Public Opinion Quarterly 61 (3), 431-451.

Noelle-Neumann, Elisabeth, 1974: The spiral of silence. A theory of public opinion, in: Journal of Communication 24 (2), 43-51. 
Noelle-Neumann, Elisabeth, 2001: Die Schweigespirale. Öffentliche Meinung unsere soziale Haut, München (6. Aufl.).

Noelle-Neumann, Elisabeth/Petersen, Thomas, 2004: The spiral of silence and the social nature of man, in: Lynda Lee Kaid (Hrsg.), Handbook of political communication research, Mahwah, 339-356.

o. V., 2013: Statistik: Wahlmonitor 2013 - Umfragen in Sendungen mit Wahlbezug, in: Media Perspektiven (12), 622.

O'Gorman, Hubert J., 1979: White and black perceptions of racial values, in: Public Opinion Quarterly 43 (1), 48-59.

Partheymüller, Julia/Schäfer, Anne, 2013: Das Informationsverhalten der Bürger im Bundestagswahlkampf 2013, in: Media Perspektiven (12), 574-588.

Petric, Gregor/Pinter, Andrej, 2002: From social perception to public expression of opinion: A structural equation modeling approach to the spiral of silence, in: International Journal of Public Opinion Research 14 (1), 37-53.

Reinemann, Carsten/Maurer, Marcus/Jandura, Olaf/Zerback, Thomas, 2013: Die Spätentscheider. Medieneinflüsse auf kurzfristige Wahlentscheidungen, Wiesbaden.

Ross, Lee/Greene, David/House, Pamela, 1977: The "false consensus effect": An egocentric bias in social perception and attribution processes, in: Journal of Experimental Social Psychology 13 (3), 279-301.

Schenk, Michael, 1995: Soziale Netzwerke und Massenmedien: Untersuchungen zum Einfluß der persönlichen Kommunikation, Tübingen.

Schenk, Michael, 2007: Medienwirkungsforschung, Tübingen (3. Aufl.).

Shamir, Jacob, 1995: Information cues and indicators of the climate of opinion: The spiral of silence theory in the Intifada, in: Communication Research 22 (1), 24-53.

Shamir, Jacob/Shamir, Michal, 2000: The anatomy of public opinion, Ann Arbor. Taylor, D. Garth, 1982: Pluralistic ignorance and the spiral of silence: A formal analysis, in: Public Opinion Quarterly 46 (3), 311.

Tsfati, Yariv, 2003: Media skepticism and climate of opinion perception, in: International Journal of Public Opinion Research 15 (1), 65-82.

Tsfati, Yariv/Stroud, Natalie Jomini/Chotiner, Adi, 2013: Exposure to ideological news and perceived opinion climate: Testing the media effects component of spiral-of-silence in a fragmented media landscape, in: The International Journal of Press/Politics 19 (1), 3-23.

Uhlaner, Carole Jean/Grofman, Bernard, 1986: The race may be close but my horse is going to win: Wish fulfillment in the 1980 presidential election, in: Political Behavior 8 (2), 101-129. 
Vallone, Robert P./Ross, Lee/Lepper, Mark R., 1985: The hostile media phenomenon: Biased perception and perceptions of media bias in coverage of the Beirut massacre, in: Journal of Personality and Social Psychology 49 (3), 577-585.

Wojcieszak, Magdalena/Price, Vincent, 2009: What underlies the false consensus effect? How personal opinion and disagreement affect perception of public opinion, in: International Journal of Public Opinion Research 21 (1), 25-46.

Zillmann, Dolf, 2002: Exemplification theory of media influence, in: Jennings Bryant/Dolf Zillmann (Hrsg.), Media effects: Advances in theory and research, Mahwah/London (2. Aufl.), 19-41.

Zillmann, Dolf, 2006: Exemplification effects in the promotion of safety and health, in: Journal of Communication 56 (1), 221-237.

Korrespondenzanschrift:

Dr. Thomas Zerback

Ludwig-Maximilians-Universität München

Institut für Kommunikationswissenschaft und Medienforschung

Oettingenstr. 67

80538 München

E-Mail: zerback@ifkw.lmu.de

Prof. Dr. Carsten Reinemann

Ludwig-Maximilians-Universität München

Institut für Kommunikationswissenschaft und Medienforschung

Oettingenstr. 67

80538 München

E-Mail: reinemann@ifkw.lmu.de

Angela Nienierza, M.A.

Ludwig-Maximilians-Universität München

Institut für Kommunikationswissenschaft und Medienforschung

Oettingenstr. 67

80538 München

E-Mail: nienierza@ifkw.lmu.de 